\title{
Strong deflection gravitational lensing by an Einstein-Lovelock ultracompact object
}

\author{
Yuan-Xing Gao ${ }^{1}, \mathbf{Y i ~ X i e}^{2, \mathrm{a}}$ (D) \\ ${ }^{1}$ School of Astronomy and Space Science, Nanjing University, Nanjing 210023, China \\ ${ }^{2}$ Purple Mountain Observatory, Chinese Academy of Sciences, Nanjing 210023, China
}

Received: 22 November 2021 / Accepted: 13 February 2022 / Published online: 22 February 2022

(C) The Author(s) 2022

\begin{abstract}
We investigate the strong deflection gravitational lensing by an Einstein-Lovelock ultracompact object. Its unique features are the relativistic images inside its photon sphere which are absent for an Einstein-Lovelock black hole. We obtain its lensing observables and evaluate their observability for the direct images of two supermassive black holes in the Galaxy and M87 respectively, Sgr A* and M87*, and for the relativistic microlensing on a star closely around $\mathrm{Sgr}$ $\mathrm{A}^{*}$. We find that although it is impossible to tell difference of the ultracompact object from the black hole in EinsteinLovelock gravity by the direct images, it might be possible to distinguish the Einstein-Lovelock ultracompact object by measuring the total flux of the relativistic microlensing in the not-so-far future.
\end{abstract}

\section{Introduction}

Detection of gravitational waves from binary black holes [16] and directly imaging the supermassive black hole M87* at the center of the giant elliptical galaxy M87 [7-12] not only reveal that black holes are abundant in the Universe, but also provide new means to study the laws of physics in the strong gravitational fields. A black hole with an event horizon and a central singularity is a fundamental object predicted by Einstein's general relativity. However, the existence of the event horizon blocks the connection between the interior and exterior regions of the black hole, resulting in Hawking radiation and the information loss problem, while the singularity straightforwardly causes the breakdown of the general relativity.

Before a quantum theory of gravity solves these problems once for all, some alternative ways might also be considered to eliminate the singularity, such as replacing it with a regular core, with a bouncing geometry, or with quasi-black

\footnotetext{
a e-mail: yixie@pmo.ac.cn (corresponding author)
}

holes (see Ref. [13] for a review and references therein). For erasing the event horizon, one might, somehow, construct an exotic compact object which is more massive than a neutron star but without the event horizon. As a subclass of the exotic compact objects, an ultracompact object is horizonless but with a photon sphere [14]. There are so many proposals for the ultracompact objects, but there are also as many as unknowns (see Ref. [15] for a review and references therein).

Recently, the 4-dimensional regularized Einstein-Lovelock theory of gravity has drawn much attention $[16,17]$. Such a regularized theory can evade the Lovelock theorem [18], which states that only the metric tensor and Einstein tensor contribute to the dynamics of 4-dimensional spacetime. Besides usual black holes in this theory [16,19-21], regular black holes are also admitted in the Einstein-Lovelock theory [22]. We find that ultracompact objects are as well permitted in such a theory. Although it is crucial for understanding the nature of these objects, the physical properties and observational signatures of the Einstein-Lovelock regular black holes and ultracompact objects are barely known.

An ultracompact object has different features from a black hole in gravitational and electromagnetic waves [15]. In gravitational-wave observation, echoes in the ringdown phase [23-27] would be a smoking-gun for the ultracompact object. However, a conclusive detection of these echoes might have to wait for next-generation gravitational waves detectors [14]. Considering recent success of radio and infrared interferometry, such as the Event Horizon Telescope (EHT) [7] and GRAVITY [28], we will focus on the behavior of the Einstein-Lovelock ultracompact object which can be accessed by the electromagnetic waves, especially signatures in the strong deflection gravitational lensing.

With a deflection angle much bigger than 1, photons in the strong deflection gravitational lensing wind more than once around the photon sphere (unstable light ring), leading to a series of relativistic images in the very close region outside the photon sphere [29] and a "shadow" inside it (see Refs. 
[30,31] for reviews and references therein). The direct image of M87* by EHT [7-12] makes it possible to observe the strong deflection gravitational lensing by a black hole, which would provide more understandings about the black holes [32-39] and a useful way for testing them [40-51]

The general character of an ultracompact object is the antiphoton sphere (stable light ring) which is located inside the photon sphere [52,53]. Under general circumstances, an asymptotically flat black hole has a photon sphere [54], while there are examples of hairy black holes with an antiphoton sphere [55] and of non-asymptotically flat black holes without any light rings [56]. As a result, besides relativistic images outside the photon sphere, the strong deflection gravitational lensing by the ultracompact object can form relativistic images inside the photon sphere [57], and might have very different observables for various objects [58-73].

Inspired by these works, we will investigate the strong deflection gravitational lensing by the Einstein-Lovelock ultracompact object and pay close attention to its signatures in its direct image and relativistic microlensing where the outer and inner relativistic images are all involved. In Sect. 2, we give a brief introduction of the 4-dimensional regularized Einstein-Lovelock gravity and its solutions of the regular black hole and ultracompact object in one special case. After analytically studying its strong deflection gravitational lensing by the Einstein-Lovelock ultracompact object in Sect. 3, we intensively examine its observables and discuss their observability for the direct images of the supermassive black holes in the Galactic Center, Sgr A*, and in the galaxy M87, M87*, in Sect. 4 and for the relativistic microlensing on a star closely around Sgr A* in Sect. 5. Finally, we conclude and discuss our results in Sect. 6.

\section{Einstein-Lovelock ultracompact object}

The Lagrangian density of the Einstein-Lovelock gravity in the $D$-dimensional spacetime is given by $(G=c=1)$ [18]

$$
\begin{aligned}
\mathcal{L}= & \frac{1}{8 \pi} \sum_{k=1}^{\bar{k}} \frac{\alpha_{k}}{2^{k} k} \delta_{\sigma_{1} \rho_{1} \ldots \sigma_{k} \rho_{k}}^{\mu_{1} v_{1} \cdots \mu_{k} v_{k}} \\
& \times R_{\mu_{1} v_{1}} \sigma_{1} \rho_{1} \ldots R_{\mu_{k} v_{k}} \sigma_{k} \rho_{k},
\end{aligned}
$$

where $\delta_{\sigma_{1} \rho_{1} \cdots \sigma_{k} \rho_{k}}^{\mu_{1} \nu_{1} \cdots \mu_{k} \nu_{k}}$ is the generalized Kronecker delta, $R_{\mu_{k} v_{k}}{ }^{\sigma_{k} \rho_{k}}$ is the Riemann tensor, $\alpha_{1}=1$ and $\alpha_{k}$ ( $k=$ $2,3, \cdots)$ are arbitrary constants. Its resulting equations of motion for the metric tensor $g_{\mu \nu}$ read [74]

$$
\begin{aligned}
0= & \sum_{k=1}^{\bar{k}} \frac{\alpha_{k}}{2^{k+1} k} \delta_{\beta \sigma_{1} \rho_{1} \ldots \sigma_{k} \rho_{k}}^{\alpha \mu_{1} \nu_{1} \cdots \mu_{k} v_{k}} \\
& \times R_{\mu_{1} v_{1}} \sigma_{1} \rho_{1} \ldots R_{\mu_{k} v_{k}} \sigma_{k} \rho_{k}
\end{aligned}
$$

Such a Lagrangian diverges as $D \rightarrow 4$, while regular terms arise in the field equations for $D \geq 3$. With the methodology proposed in Refs. [16,17,75], a 4-dimensional static and spherically symmetric spacetime with mass $m$ • can be found as

$\mathrm{d} s^{2}=-A(r) \mathrm{d} t^{2}+B(r) \mathrm{d} r^{2}+C(r)\left(\mathrm{d} \theta^{2}+\sin ^{2} \mathrm{~d} \phi^{2}\right)$,

where $A(r)$ is defined through a new variable $\psi(r)$ as

$A(r)=[B(r)]^{-1}=1-r^{2} \psi(r)$,

$C(r)=r^{2}$,

and $\psi(r)$ satisfies the algebraic equation

$P[\psi(r)] \equiv \sum_{k=1}^{\bar{k}} \tilde{\alpha}_{k} \psi^{k}=\frac{2 m \bullet}{r^{3}}$,

where

$\tilde{\alpha}_{k}=\frac{\alpha_{k}}{k} \prod_{p=1}^{2 k-2}(D-2-p)$.

In order to solve the metric tensor (3) from the field Eq. (2), the expression of $P[\psi(r)]$ must be specified. With the choice of $\bar{k}=+\infty$ that was first proposed in Ref. [76] and with the assumption adopt in Ref. [22] that $P[\psi(r)]$ could be taken and expanded as

$$
\begin{aligned}
P[\psi(r)] & =\frac{1}{\eta_{\bullet}} \arcsin \left(\eta_{\bullet} \psi\right) \\
& =\psi+\frac{1}{6} \eta_{\bullet}^{2} \psi^{3}+\frac{3}{40} \eta_{\bullet}^{4} \psi^{5}+\cdots
\end{aligned}
$$

where the parameter $\eta_{\bullet}$ with the dimension of [length $]^{2}$ has to be introduced to balance the dimension of Eq. (6), the explicit form of $\psi$ is, therefore, found as [22]

$\psi(r)=\frac{1}{\eta_{\bullet}} \sin \left(\frac{2 \eta_{\bullet} m_{\bullet}}{r^{3}}\right)$.

By comparing Eq. (8) with Eq. (6), one can obtain that

$\tilde{\alpha}_{1}=1, \tilde{\alpha}_{2}=0, \tilde{\alpha}_{3}=\frac{\eta_{\bullet}^{2}}{6}, \tilde{\alpha}_{4}=0, \tilde{\alpha}_{5}=\frac{3}{40} \eta_{\bullet}^{4}, \ldots$

When $\eta_{\bullet}=0$, the metric (3) returns to the one of the Schwarzschild black hole. As $\eta_{\bullet} \leftrightarrow-\eta_{\bullet}, A(r)$ remains unchanged so that we can simply focus on $\eta_{\bullet} \geq 0$. The spacetime (3) is asymptotically flat as $r \rightarrow+\infty$, while $A(r)$ is finite and unity as $r \rightarrow 0$. In order to ensure the existence of the event horizon(s) that $[B(r)]^{-1}=A(r)=0$ has real and positive $\operatorname{root}(\mathrm{s})$, we find that the dimensionless parameter

$\eta \equiv \frac{\eta \bullet}{m_{\bullet}^{2}}$

must belong to the domain

$\mathcal{D}_{\mathrm{RBH}}=\left\{\eta \mid 0<\eta \leq \eta_{\mathrm{H}} \approx 2.38617\right\}$, 
so that the spacetime (3) describes a regular black hole. Otherwise, it represents a horizonless object if $\eta_{\bullet}>\eta_{\mathrm{H}}$.

Based on the geodesic motion of a photon in the equator of the spacetime (3), its radial equation of motion is

$\dot{r}^{2}+L^{2} V_{\mathrm{eff} \bullet}(r)=E^{2}$,

where $A(r) B(r)=1$ is used, $E$ and $L$ are respectively the energy and angular momentum of the photon, and the effective potential per $L^{2}$ is

$V_{\mathrm{eff} \bullet}(r)=\frac{A(r)}{C(r)}$.

An unstable circular light ring, which is also called the photon sphere, exists as long as the conditions that $V_{\mathrm{eff}}^{\prime}\left(r_{\mathrm{m}}\right)=0$ and $V_{\text {eff }}^{\prime \prime}\left(r_{\mathrm{m}}\right)<0$ are satisfied for a positive radius $r_{\mathrm{m}}$ where ' denotes the derivative against $r$. When $\eta_{\bullet}=0, r_{\mathrm{m}}$ returns to its value for the Schwarzschild black hole $r_{\mathrm{m}, \mathrm{Sch}}=3 \mathrm{~m}_{\bullet}$. The radius $r_{\text {sh }}$ of the gravitationally lensed photon sphere as observed at infinity is [77]

$r_{\mathrm{sh}}=\frac{r_{\mathrm{m}}}{\sqrt{A\left(r_{\mathrm{m}}\right)}}$.

When $\eta_{\bullet}=0, r_{\text {sh }}$ goes back to the one of the Schwarzschild black hole $r_{\text {sh,Sch }}=3 \sqrt{3} m_{\bullet}$. This radius casts a shadow for a black hole. However, such a "shadow" might no longer be applicable for a ultracompact object, since there is no event horizon and consequently no photon absorption. For later convenience, we define the following dimensionless quantities that

$x=\frac{r}{m_{\bullet}}, \quad x_{\mathrm{m}}=\frac{r_{\mathrm{m}}}{m_{\bullet}}, \quad x_{\mathrm{sh}}=\frac{r_{\mathrm{sh}}}{m_{\bullet}}$,

and

$$
\begin{aligned}
V_{\mathrm{eff}}(x) & =m_{\bullet}^{2} V_{\mathrm{eff} \bullet}(x), \\
V_{\mathrm{eff}, x}(x) & =m_{\bullet}^{3} V_{\mathrm{eff} \bullet}^{\prime}(r)=\frac{2}{x^{4}} P(x),
\end{aligned}
$$

where

$P(x)=3 \cos \left(\frac{2 \eta}{x^{3}}\right)-x$.

Since $V_{\mathrm{eff} \bullet}^{\prime}\left(r_{\mathrm{m}}\right)=0$ is equivalent to $P\left(x_{\mathrm{m}}\right)=0$ for any positive $r_{\mathrm{m}}$ and $x_{\mathrm{m}}$, we can immediately obtain that $x_{\mathrm{m}}$ is no more than 3 .

We find that there is an interesting feature about the (dimensionless) radius of the photon sphere. It suddenly jumps from $x_{\mathrm{m}} \approx 2.56210$ down to $x_{\mathrm{m}} \approx 1.21506$ as $\eta$ increases and crosses $\eta_{\mathrm{U}} \approx 4.60077$, because the oscillating nature of the sine function in $A(r)$. Figure 1 shows such a jump of $P(x)$ for two cases with $\eta=4.2$ and $\eta=5.2$ where the black dots denote their dimensionless radii of the photon spheres $x_{\mathrm{m}}$. The radius of the gravitationally lensed photon sphere shares the same behavior due to its directly connection with the photon sphere through Eq. (15). The top and middle

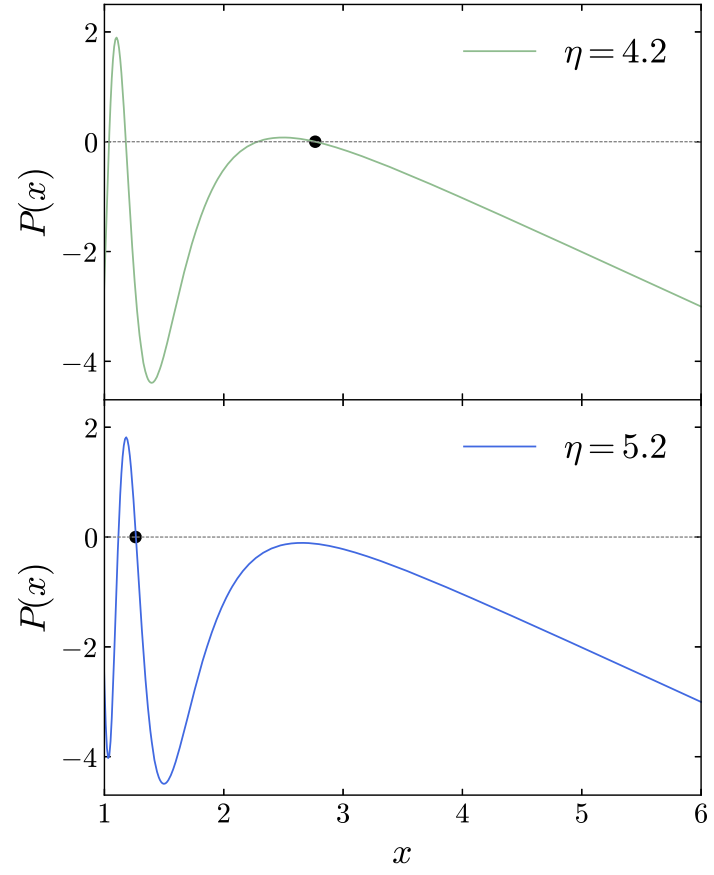

Fig. $1 P(x)$ for $\eta=4.2$ (top) and $\eta=5.2$ (bottom) are shown. The dimensionless radius of the photon sphere $x_{\mathrm{m}}$ (denoted by black dot) jumps from about 2.5 (top) to about 1.2 (bottom)

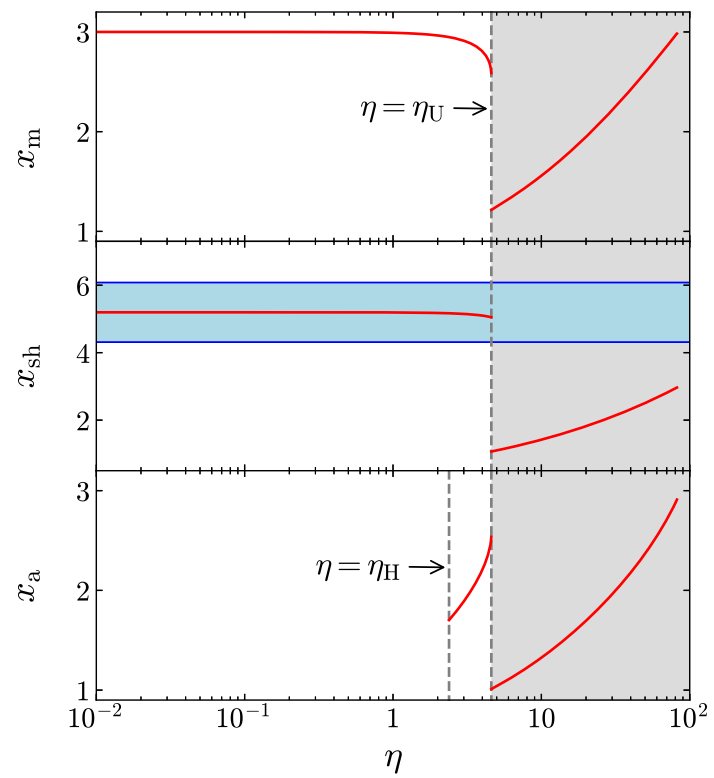

Fig. 2 The (dimensionless) radii of the photon sphere $x_{\mathrm{m}}$ (top), the gravitationally lensed photon sphere $x_{\mathrm{sh}}$ (middle) and the antiphoton sphere $x_{\mathrm{a}}$ (bottom) with respect to $\eta$. The blue shadowed region of $x_{\mathrm{sh}}$ is consistent with the EHT observation [78], while the grey shadowed domain of $\eta$ is ruled out

panel of Fig. 2 demonstrate $x_{\mathrm{m}}$ and $x_{\mathrm{sh}}$ with respect to $\eta$ and their sudden changes near $\eta=\eta_{\mathrm{U}}$. However, this theoretical behavior is not supported by practice. Based on the direct image of M87* [7-12], it was found [78] that the size of the 


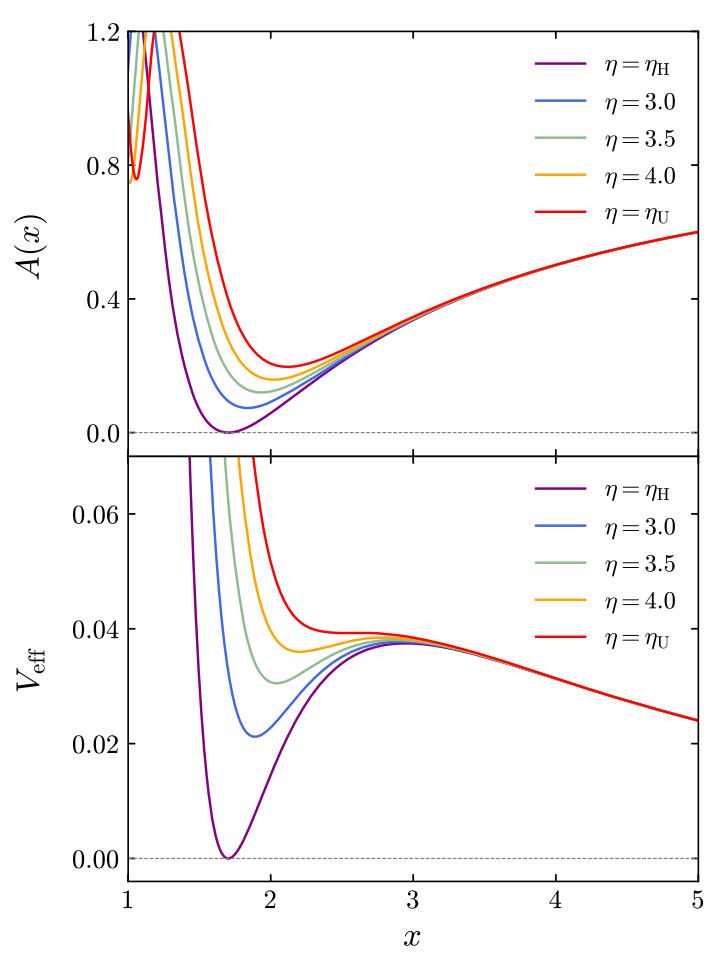

Fig. $3 A(x)$ (top) and $V_{\text {eff }}$ (bottom) with respect to $x$ fro some $\eta$

M87*'s shadow is consistent within $17 \%$ at $68 \%$ confidence level with the predicted size of the general relativity. Following the approach of Ref. [78], we obtain a preliminary bound on (dimensionless) $\eta$ as $0 \leq \eta<\eta_{\mathrm{U}}$ and rule out the domain of $\eta \geq \eta_{\mathrm{U}}$ by EHT observation as the middle panel of Fig. 2 shown since its $x_{\mathrm{sh}}$ is outside the allowable area given by EHT. Here, we do not take the spin of the central compact object and its inclination into account, whereas their overall influence on the size and shape of the shadow is less than $4 \%$ and $7 \%$, respectively [79]. We think that this bound on $\eta$ might be valid at the leading order and the EHT measurement might be adequately adopted for testing the irrational spacetime (3). According to Eq. (11), $\eta_{\bullet}$ for various systems could still be distinct even if $\eta$ is fixed, since their $m_{\bullet}$ are different.

Meanwhile, a stable circular light ring, which is called the antiphoton sphere [57], exists if $V_{\text {eff }}^{\prime}\left(r_{\mathrm{a}}\right)=0$ and $V_{\mathrm{eff} \bullet}^{\prime \prime}\left(r_{\mathrm{a}}\right)<0$ for a positive radius $r_{\mathrm{a}}$. The antiphoton sphere starts to emerge when $\eta$ grows over $\eta_{\mathrm{H}}$. As $\eta$ increases further, the photon and antiphoton spheres will become closer to each other and eventually disappear at $\eta=\eta_{\mathrm{U}}$ such that $V_{\mathrm{eff} \bullet}^{\prime \prime}=0$. Since the domain of $\eta>\eta_{\mathrm{U}}$ is disfavored by the EHT observation on M87* [78], we will not consider its antiphoton sphere. Therefore, a horizonless spacetime (3) with both photon and antiphoton spheres is called an Einstein-Lovelock ultracompact object by demanding that

$\mathcal{D}_{\mathrm{UCO}}=\left\{\eta \mid \eta_{\mathrm{H}}<\eta<\eta_{\mathrm{U}}\right\}$.
Figure 3 displays the variation of $A(x)$ and $V_{\text {eff }}$ for some $\eta \in \mathcal{D}_{\text {UCO }}$ respectively in the top and bottom panels. For an Einstein-Lovelock ultracompact object, it has no event horizon that $A(x)=0$ has no roots, and it has the photon and antiphoton spheres that $V_{\text {eff }}$ has the minimum and maximum points until they merger into one as $\eta \rightarrow \eta_{\mathrm{U}}$. Due to the oscillating behavior of $A(r)$, which can be seen in the upperleft corner of the top panel of Fig. 3, $V_{\text {eff }} \propto A(r)$ does have other extrema besides those shown in the bottom panel of Fig. 3, also see Fig. 1. In order to more clearly show this outermost pair of extrama of $V_{\text {eff }}$ and their change with respect to $\eta$, we choose to zoom in this sub-figure and keep other extrema out. Such an outermost pair can generate the most significant inner and outer relativistic images, since they have the smallest $V_{\text {eff }}$ and the biggest impact parameters, which are proportional to $V_{\text {eff }}^{-1 / 2}$ (see Sect. 3 for details). We only consider these leading lensing effects of the outermost pair in this work and leave other subtle effects in our next move. The bottom panel of Fig. 2 shows the (dimensionless) radius $x_{\mathrm{a}}$ of the antiphoton sphere with respect to $\eta$ where we define

$x_{\mathrm{a}}=\frac{r_{\mathrm{a}}}{m_{\bullet}}$.

In the following sections, we will investigate the strong deflection gravitational lensing by an Einstein-Lovelock ultracompact object and detailedly analyse its observables.

\section{Strong deflection gravitational lensing}

In the spacetime (3) as a lens, the path of a photon emitted would be deflected by an angle as [80]

$\hat{\alpha}\left(r_{0}\right)=2 \int_{r_{0}}^{\infty} \frac{\mathrm{d} r}{r \sqrt{\left[r^{2} u^{-2}-A(r)\right]}}-\pi$,

where $A(r) B(r)=1$ is used, $r_{0}$ is the closest approach of the photon and the impact parameter $u$ is

$u=\frac{r_{0}}{\sqrt{A\left(r_{0}\right)}}$.

When $r_{0} \gg m_{\bullet}$, the deflection angle $\hat{\alpha}$ will be much less than 1 , resulting in the weak deflection gravitational lensing. When $r_{0}$ approaches to $r_{\mathrm{m}}, \hat{\alpha}$ will increase and eventually explode. In order to characterize the relation among the images, the source, the lens and the observer, we take the lensing equation as $[32,33]$

$\tan \mathcal{B}=\tan \vartheta-\frac{D_{\mathrm{LS}}}{D_{\mathrm{OS}}}[\tan \vartheta+\tan (\hat{\alpha}-\vartheta)]$,

where the angular positions of the source and the lensed images are respectively denoted by $\mathcal{B}$ and $\vartheta$, and the distances from the source to the lens and to the observer are respectively indicated by $D_{\mathrm{LS}}$ and $D_{\mathrm{OS}}$. The magnification $\mu$ 
is defined as the flux ratio of the lensed image to the unlensed source, which reads [81]

$\mu=\left|\frac{\sin \mathcal{B}}{\sin \vartheta} \frac{\mathrm{d} \mathcal{B}}{\mathrm{d} \vartheta}\right|^{-1}$.

Before we proceed, it is worth mentioning that the Schwarzschild black hole, the Einstein-Lovelock regular black hole and ultracompact object have the same Taylor expansions of $A(r)$ and $B(r)$ in terms of $m_{\bullet} r^{-1}$ up to the order of $\mathcal{O}\left(m_{\bullet}^{6} r^{-6}\right)$. Therefore, these spacetimes have indistinguishable signatures in the weak deflection gravitational lensing, such as the deflection angle, the positions and magnification of images, and the centroid (see Appendix A for details). It also suggests that the strong deflection gravitational lensing might be the way to tell their difference.

In the scenario of the strong deflection gravitational lensing by an Einstein-Lovelock ultracompact object, the divergence of the deflection angle generates a set of infinite relativistic images outside and inside the photon sphere. For a photon with $r_{0}>r_{\mathrm{m}}$, it can form a relativistic image outside the photon sphere and its deflection angle in the strong deflection limit is $[34,38]$

$$
\begin{aligned}
\hat{\alpha}_{+}(u)= & -\bar{a}_{+} \log \left(\frac{u}{u_{\mathrm{m}}}-1\right)+\bar{b}_{+} \\
& +\mathcal{O}\left[\left(u-u_{\mathrm{m}}\right) \log \left(u-u_{\mathrm{m}}\right)\right],
\end{aligned}
$$

where and hereafter a quantity with a subscript "m" means its value at the photon sphere $r_{\mathrm{m}}$, e.g., $u_{\mathrm{m}}=u\left(r_{\mathrm{m}}\right)$. The coefficients of the strong deflection limit in Eq. (32) are [38]

$\bar{a}_{+}=\sqrt{\frac{2}{C_{\mathrm{m}}^{\prime \prime} A_{\mathrm{m}}-C_{\mathrm{m}} A_{\mathrm{m}}^{\prime \prime}}}$,

$\bar{b}_{+}=-\pi+\bar{a}_{+} \log \left[r_{\mathrm{m}}^{2}\left(\frac{C_{\mathrm{m}}^{\prime \prime}}{C_{\mathrm{m}}}-\frac{A_{\mathrm{m}}^{\prime \prime}}{A_{\mathrm{m}}}\right)\right]+I_{+}\left(r_{\mathrm{m}}\right)$,

where

$$
\begin{aligned}
I_{+}\left(r_{\mathrm{m}}\right) & =2 \int_{0}^{1}\left[\frac{r_{\mathrm{m}}}{(1-z)^{2}} \sqrt{\frac{C_{\mathrm{m}}}{\left(A_{\mathrm{m}} C_{z}-A_{z} C_{\mathrm{m}}\right) C_{z}}}-\frac{\bar{a}_{+}}{|z|}\right] \mathrm{d} z, \\
z & =1-\frac{r_{\mathrm{m}}}{r},
\end{aligned}
$$

and a quantity $F$ with subscript " $z$ " means

$$
F_{z} \equiv F[r(z)]=F\left[r_{\mathrm{m}}(1-z)^{-1}\right] \text {. }
$$

For a photon with $r_{0}<r_{\mathrm{m}}$, it will generate a relativistic image inside the photon sphere and its deflection angle in the strong deflection limit is [57]

$$
\begin{aligned}
\hat{\alpha}_{-}(u)= & -\bar{a}_{-} \log \left(\frac{u_{\mathrm{m}}^{2}}{u^{2}}-1\right)+\bar{b}_{-} \\
& +\mathcal{O}\left[\left(u_{\mathrm{m}}^{2}-u^{2}\right) \log \left(u_{\mathrm{m}}^{2}-u^{2}\right)\right],
\end{aligned}
$$

where

$$
\begin{aligned}
\bar{a}_{-}= & 2 \bar{a}_{+}, \\
\bar{b}_{-}= & 2 \bar{a}_{+} \log \left[2 r_{\mathrm{m}}^{2}\left(\frac{C_{\mathrm{m}}^{\prime \prime}}{C_{\mathrm{m}}}-\frac{A_{\mathrm{m}}^{\prime \prime}}{A_{\mathrm{m}}}\right)\left(\frac{r_{\mathrm{m}}}{r_{\mathrm{c}}}-1\right)\right] \\
& +I_{-}\left(r_{\mathrm{c}}\right)-\pi \\
I_{-}\left(r_{\mathrm{c}}\right)= & 2 \int_{1-\frac{r_{\mathrm{m}}}{r_{\mathrm{c}}}}^{1}\left[\frac{r_{\mathrm{m}}}{(1-z)^{2}} \sqrt{\frac{C_{\mathrm{c}}}{\left(A_{\mathrm{c}} C_{z}-A_{z} C_{\mathrm{c}}\right) C_{z}}}-\frac{\bar{a}_{+}}{|z|}\right] \mathrm{d} z .
\end{aligned}
$$

Here $r_{\mathrm{c}}$ satisfies the equation $V_{\mathrm{eff}}\left(r_{\mathrm{c}}\right)=V_{\mathrm{eff}}\left(r_{\mathrm{m}}\right)$ and the condition $r_{\mathrm{c}}<r_{\mathrm{m}}$; and a quantity with subscript "c" denotes its value at $r_{\mathrm{c}}$.

In the strong deflection gravitational lensing, we assume that the source, the lens and the observer are in a nearly collinear alignment so that the lens Eq. (24) can be simplified as [82]

$\mathcal{B}=\vartheta-\frac{D_{\mathrm{LS}}}{D_{\mathrm{OS}}}[\hat{\alpha}(\vartheta)-2 n \pi], \quad n \in \mathbb{Z}^{+}$.

For the outer relativistic images, their deflection angles have $\hat{\alpha}(\vartheta)=\hat{\alpha}_{+}(u)$ where $u=\vartheta D_{\mathrm{OL}}$, and the outer relativistic Einstein rings $\vartheta_{+n}^{\mathrm{E}}$ with $\alpha_{+}=2 n \pi$ are located at

$\vartheta_{+n}^{\mathrm{E}}=\frac{u_{m}}{D_{\mathrm{OL}}}\left(1+e_{+n}\right)$,

where

$e_{+n}=\exp \left(\frac{\bar{b}_{+}-2 n \pi}{\bar{a}_{+}}\right)$

As $n \rightarrow \infty$, we can have the apparent angular size of the photon sphere as

$\theta_{\infty} \equiv \vartheta_{+\infty}^{\mathrm{E}}=\frac{u_{m}}{D_{\mathrm{OL}}}$

The outer relativistic images $\vartheta_{+n}$ are located at [34]

$\vartheta_{+n}=\vartheta_{+n}^{\mathrm{E}}+\frac{u_{m} e_{+n} D_{\mathrm{OS}}}{\bar{a}_{+} D_{\mathrm{OL}} D_{\mathrm{LS}}}\left(\mathcal{B}-\vartheta_{+n}^{\mathrm{E}}\right)$,

and their magnification based on (25) are [34]

$\mu_{+n}=\frac{u_{m}^{2} D_{\mathrm{OS}}}{\bar{a}_{+} \mathcal{B} D_{\mathrm{OL}}^{2} D_{\mathrm{LS}}}\left(1+e_{+n}\right) e_{+n}$.

For the inner relativistic images, their deflection angles have $\hat{\alpha}(\vartheta)=\hat{\alpha}_{-}(u)$, and the inner relativistic Einstein rings $\vartheta_{-n}^{\mathrm{E}}$ with $\alpha_{-}=2 n \pi$ are located at [57]

$\vartheta_{-n}^{\mathrm{E}}=\frac{u_{m}}{D_{\mathrm{OL}} \sqrt{1+e_{-n}}}$,

where

$e_{-n}=\exp \left(\frac{\bar{b}_{-}-2 n \pi}{\bar{a}_{-}}\right)$. 
As $n \rightarrow \infty$, we can have the apparent angular size of the antiphoton sphere as

$\vartheta_{-\infty}^{\mathrm{E}}=\frac{u_{m}}{D_{\mathrm{OL}}}=\theta_{\infty}$,

which is exactly the same as the one of the photon sphere. The inner relativistic images $\vartheta_{-n}$ are located at [57]

$\vartheta_{-n}=\vartheta_{-n}^{\mathrm{E}}-\frac{u_{m} e_{-n} D_{\mathrm{OS}}}{2 \bar{a}_{-} D_{\mathrm{OL}} D_{\mathrm{LS}}} \frac{\left(\mathcal{B}-\vartheta_{-n}^{\mathrm{E}}\right)}{\left(1+e_{-n}\right)^{3 / 2}}$,

and their magnification are [57]

$\mu_{-n}=\frac{u_{m}^{2} D_{\mathrm{OS}}}{2 \bar{a}_{-} \mathcal{B} D_{\mathrm{OL}}^{2} D_{\mathrm{LS}}} \frac{e_{-n}}{\left(1+e_{-n}\right)^{2}}$.

With these positions and magnification of the outer and inner relativistic images, we will intensively discuss the observables of the direct imaging of the supermassive black holes Sgr A* and M87* and of the relativistic microlensing by Sgr A*.

\section{Direct images of Sgr A* and M87*}

Since it is almost impossible to distinguish an ultracompact object from a black hole through gravitational wave [15], we might assume that Sgr A* and M87* would be either the Einstein-Lovelock regular black holes or the EinsteinLovelock ultracompact objects respectively with the mass $M_{\mathrm{SgrA} *}=4.28 \times 10^{6} M_{\odot}$ and the distance $D_{\mathrm{OL}, \mathrm{SgrA} *}=8.32$ $\mathrm{kpc}[83]$ and with $M_{\mathrm{M} 87 *}=6.5 \times 10^{9} M_{\odot}$ and $D_{\mathrm{OL}, \mathrm{M} 87 *}=$ 16.9 Mpc [12].

If the innermost and outermost relativistic images could only be separated and all of the others would be packed together at the photon sphere $\theta_{\infty}$, the separations among these relativistic images can obtained as

$s_{i, j} \equiv \vartheta_{i}-\vartheta_{j}$,

and their differences in brightness can be found as

$\Delta m_{i, j} \equiv 2.5 \log _{10}\left(\frac{\mu_{i}}{\mu_{j}}\right)$,

where $i, j=-1, \infty, 1$ are respectively taken for the innermost relativistic image, the photon sphere and the outermost one. With the help of $u_{\mathrm{m}} \ll D_{\mathrm{OL}}$ for Sgr A* and M87*, we can simplify these observables as

$$
\begin{aligned}
s_{+1, \infty} & =\theta_{\infty} e_{+1}, \\
s_{\infty,-1} & =\theta_{\infty}\left[1-\left(1+e_{-1}\right)^{-1 / 2}\right], \\
s_{+1,-1} & =\theta_{\infty}\left[1+e_{+1}-\left(1+e_{-1}\right)^{-1 / 2}\right], \\
\Delta m_{+1, \infty} & =2.5 \log _{10}\left[\left(\mu_{\mathrm{sh}}\right)^{-1} e_{+1}\left(1+e_{+1}\right)\right], \\
\Delta m_{-1, \infty} & =-2.5 \log _{10}\left[4 \mu_{\mathrm{sh}}\left(e_{-1}\right)^{-1}\left(1+e_{-1}\right)^{2}\right], \\
\Delta m_{+1,-1} & =2.5 \log _{10}\left[4 \frac{e_{+1}}{e_{-1}}\left(1+e_{+1}\right)\left(1+e_{-1}\right)^{2}\right],
\end{aligned}
$$

where the brightness of the packed at the photon sphere contributed by the inner and outer relativistic images so that its magnification reads

$$
\begin{aligned}
\mu_{\mathrm{sh}} & =\sum_{j=2}^{\infty}\left(\mu_{+j}+\mu_{-j}\right) \\
& =\sum_{j=2}^{\infty}\left[\left(1+e_{+j}\right) e_{+j}+\frac{e_{-j}}{4\left(1+e_{-j}\right)^{2}}\right] .
\end{aligned}
$$

The angular separation between the outermost relativistic image and the photon sphere is denoted as $s_{+1, \infty}$, the one between the photon sphere and the innermost relativistic image is indicated as $s_{\infty,-1}$, and the one between the outermost and innermost images is denoted as $s_{+1,-1}$. The brightness differences among these images are respectively $\Delta m_{+1, \infty}, \Delta m_{-1, \infty}$ and $\Delta m_{+1,-1}$. Although all of the angular separations are proportional to the apparent size of the photon sphere which relies on the mass $m$ • and the distance $D_{\mathrm{OL}}$, their brightness differences are independent of these two properties of the lens.

From top to bottom, Fig. 4 shows the angular observables $\theta_{\infty}, s_{+1, \infty}, s_{\infty,-1}$ and $s_{-1,+1}$ for the Einstein-Lovelock regular black hole (if applicable) on the domain $\mathcal{D}_{\mathrm{RBH}}$ and for the Einstein-Lovelock ultracompact object on the domain $\mathcal{D}_{\text {UCO }}$ in the cases of Sgr A* and M87*. The apparent radius of the photon sphere $\theta_{\infty}$ shrinks as $\eta$ increases as shown in the top panel of Fig. 4. It means that the Einstein-Lovelock regular black hole has a smaller $\theta_{\infty}$ than the Schwarzschild black hole (when $\eta=0$ ), while $\theta_{\infty}$ of the Einstein-Lovelock ultracompact object will be the smallest. For Sgr A*, $\theta_{\infty}$ decreases from $26.3 \mu$ as to $26.2 \mu$ as on $\mathcal{D}_{\mathrm{RBH}}$, and it continues to descend to $25.6 \mu$ as on $\mathcal{D}_{\text {UCO }}$. For M87*, $\theta_{\infty}$ decreases from $19.7 \mu$ as to $19.6 \mu$ as on $\mathcal{D}_{\mathrm{RBH}}$ and reduces from 19.6 $\mu$ as to $19.1 \mu$ as on $\mathcal{D}_{\text {UCO }}$. We find that the apparent sizes of the photon sphere $\theta_{\infty}$ of the Einstein-Lovelock regular black hole and ultracompact object can be observed with EHT, while their deviation from the one of Schwarzschild black hole are no more than $0.7 \mu$ as, which is beyond the current ability of EHT [7]. Therefore, it is impossible to distinguish the Schwarzschild black hole, the Einstein-Lovelock regular black hole and the Einstein-Lovelock ultracompact object solely based on the apparent size of the photon sphere of Sgr A* and $\mathrm{M}^{*} 7^{*}$.

From the second panel of Fig. 4, we can find that as $\eta$ increases, the angular separation between the outermost image and other packaged images on the photon sphere $s_{+1, \infty}$ increases monotonically on $\mathcal{D}_{\mathrm{RBH}}$, while it grows further to a peak and decreases on $\mathcal{D}_{\mathrm{UCO}}$. For $\mathrm{Sgr} \mathrm{A}^{*}, s_{+1, \infty}$ on $\mathcal{D}_{\mathrm{RBH}}$ can reach about 42 nanoarcsecond (nas), which is 1.25 times bigger than the one of the Schwarzschild black hole (in the case of $\eta=0$ ), and it can arrive at the peak of 95 nas on $\mathcal{D}_{\text {UCO }}$. For M87*, $s_{+1, \infty}$ is at the level of 31 nas on $\mathcal{D}_{\mathrm{RBH}}$ 


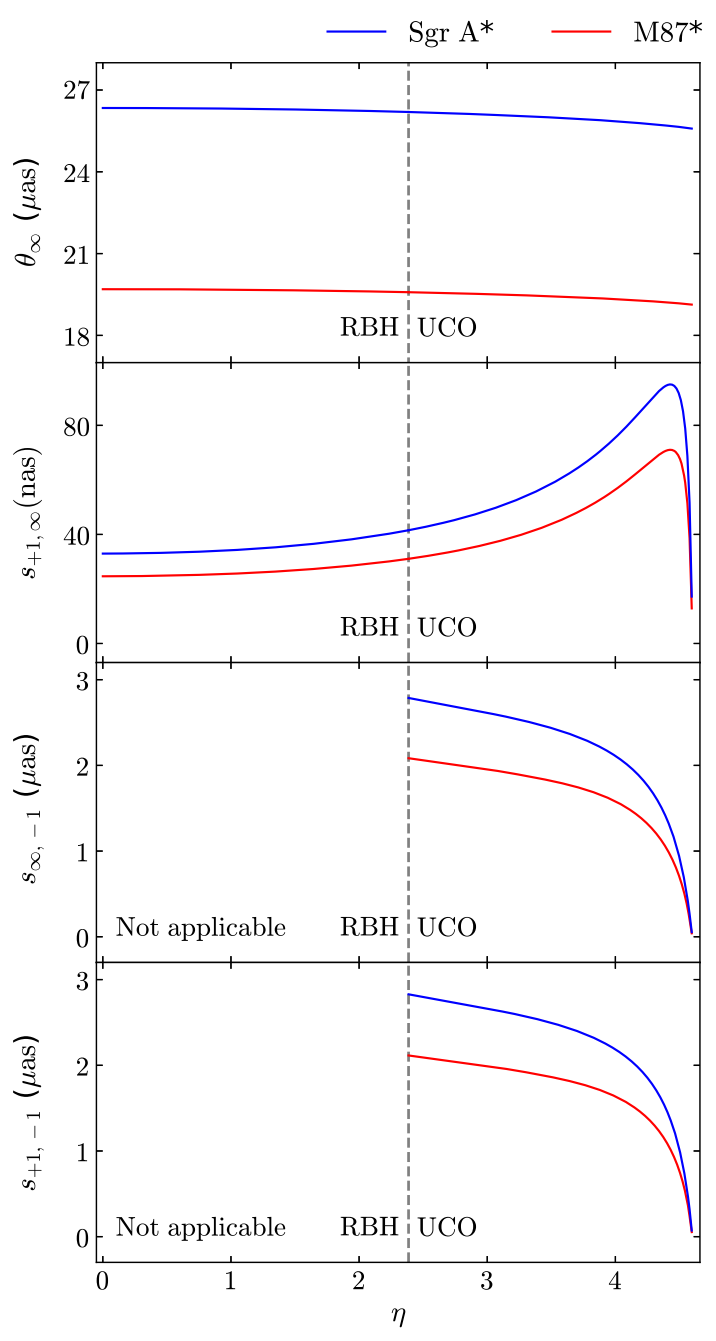

Fig. 4 The apparent radius of the photon sphere $\theta_{\infty}$ and the angular separations between relativistic images $s_{+1, \infty}, s_{\infty,-1}$ and $s_{+1,-1}$ are shown with respect to $\eta$ for an Einstein-Lovelock regular black hole (if applicable) and for an Einstein-Lovelock ultracompact object in the cases of Sgr A* (blue) and M87* (red)

and its peak on $\mathcal{D}_{\mathrm{UCO}}$ is about 71 nas. Although $s_{+1, \infty}$ has very different trends on $\mathcal{D}_{\mathrm{UCO}}$ from those on $\mathcal{D}_{\mathrm{RBH}}$, its values on these two domains are too tiny to resolve, meaning that it is unable to distinguish the Schwarzschild black hole, Einstein-Lovelock regular black hole and Einstein-Lovelock ultracompact object through measurement on $s_{+1, \infty}$ in the near future.

The third and forth panels of Fig. 4 show the angular separations between the packaged images on the photon sphere and the innermost image $s_{\infty,-1}$ and between the outermost image and the innermost one $s_{+1,-1}$, respectively, both of which are unique observables for the Einstein-Lovelock ultracompact object since the Einstein-Lovelock regular black hole has no inner relativistic images inside the photon sphere. They decrease monotonically as the increment of $\eta$ on $\mathcal{D}_{\mathrm{UCO}}$. Moreover, $s_{+1,-1}$ and $s_{\infty,-1}$ share almost the

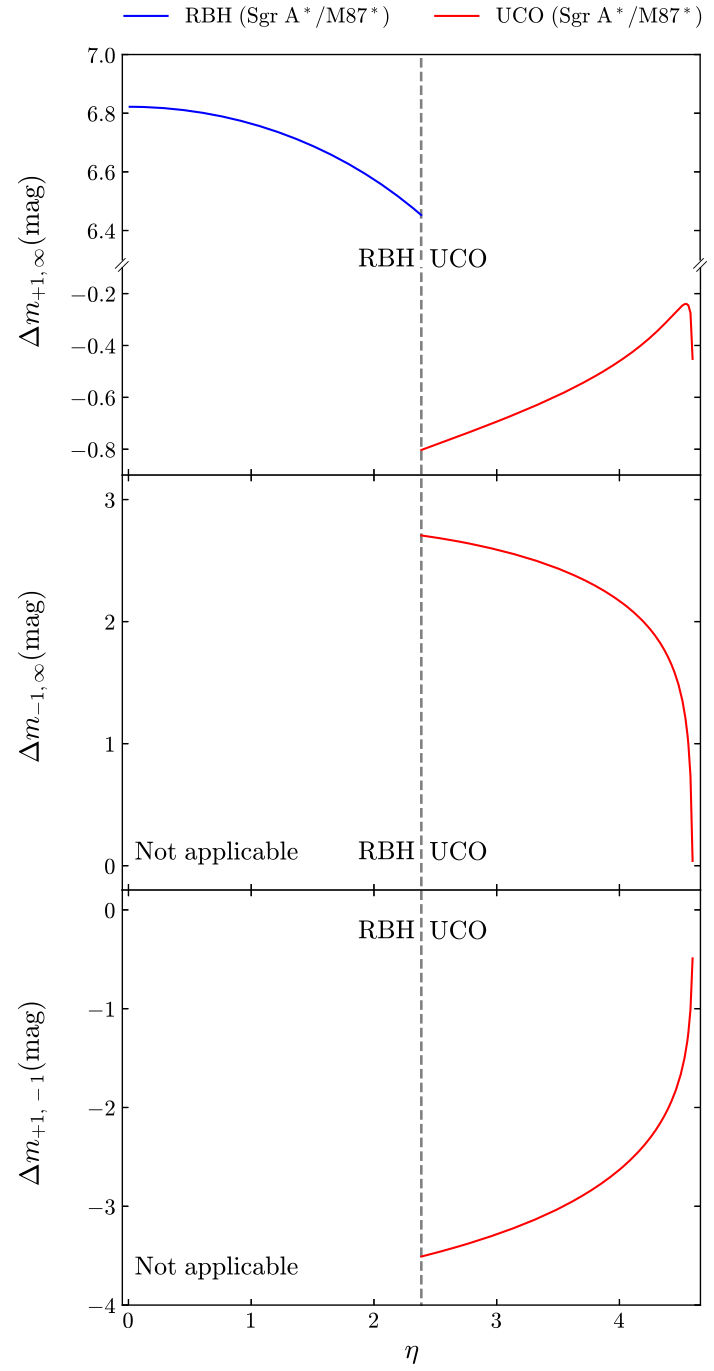

Fig. 5 The brightness differences $\Delta m_{+1, \infty}, \Delta m_{-1, \infty}$ and $\Delta m_{+1,-1}$ with respect to $\eta$ for an Einstein-Lovelock regular black hole and for an Einstein-Lovelock ultracompact object in the cases of SgrA* and M87*

same shape since their difference $s_{+1,-1}-s_{\infty,-1}=s_{+1, \infty}$ is significantly less than both of them. For Sgr A*, $s_{\infty,-1}$ and $s_{+1,-1}$ reduce from about $2.8 \mu$ as to about $0.8 \mu$ as; and for $\mathrm{M} 87^{*}$, they decrease from about $2.0 \mu$ as to about 0.8 $\mu$ as. Although $s_{\infty,-1}$ and $s_{+1,-1}$ can reach the level of a few $\mu$ as which is at least 20 times bigger than $s_{+1, \infty}$, they are close to but still beyond the current resolution of EHT [7]. It also suggests that it is not feasible to tell difference of the Einstein-Lovelock ultracompact object by its inner relativistic images.

Figure 5 shows the brightness differences between the outermost image and other packaged images on the photon sphere $\Delta m_{+1, \infty}$, between the innermost image and the packaged images $\Delta m_{-1, \infty}$, and between the outermost and innermost ones $\Delta m_{+1,-1}$ from top to bottom, respectively. Since these observables depend on $\eta$ only, instead of the mass and 
the distance of the lens, Sgr A* and M87* have the same curves of them. From the top panel of Fig. 5, $\Delta m_{+1, \infty}$ on $\mathcal{D}_{\mathrm{RBH}}$ indicates that the outermost image is brighter than the packaged images by about $6.4-6.8$ mag. However, $\Delta m_{+1, \infty}$ on $\mathcal{D}_{\text {UCO }}$ suggests that the outermost image is fainter than the packaged images by about $0.2-0.8 \mathrm{mag}$, because the inner relativistic images of the ultracompact object on photon sphere brighten the packaged images. These two totally different behaviors provide a straightforward way to distinguish an Einstein-Lovelock ultracompact object from an EinsteinLovelock regular black hole as long as the outermost relativistic image can be separated. The middle and bottom panels of Fig. 5 show $\Delta m_{-1, \infty}$ and $\Delta m_{+1,-1}$, both of which are unique features of the inner relativistic images of an ultracompact object. $\Delta m_{-1, \infty}$ on $\mathcal{D}_{\text {UCO }}$ indicates that the innermost relativistic image is brighter than the packaged images on the photon sphere by about $0.5-2.7 \mathrm{mag}$, while $\Delta m_{+1,-1}$ on $\mathcal{D}_{\text {UCO }}$ means that the outermost relativistic image is fainter than the innermost one by about 0.5-3.5 mag. Nevertheless, the angular separation between these relativistic images are too small to resolve for current ground based radio telescopes array, making it impossible to detecting and testing the Einstein-Lovelock ultracompact object.

In a summary, after analysing the angular separations and brightness differences between the inner and outer relativistic images, we find that (1) both the angular separation and the brightness of the innermost relativistic image of the Einstein-Lovelock ultracompact object are more significant than those of the outermost relativistic one; (2) the observables in the strong deflection gravitational lensing by the Schwarzschild black hole, the Einstein-Lovelock regular black hole on $\mathcal{D}_{\mathrm{RBH}}$ and Einstein-Lovelock ultracompact object on $\mathcal{D}_{\mathrm{UCO}}$ are all consistent with the measured size of M87*'s shadow by EHT; and (3) it makes these three spacetimes indistinguishable currently due to the lack of higher angular resolution.

This situation triggers us to look for some gravitational lensing observables without resolving the lensed images.

\section{Relativistic microlensing}

In the scenario of the weak deflection gravitational lensing by a point mass, two lensed images are formed. When these two images are unable to be resolved, it leads to microlensing with two fundamental observables that are the total magnification and the centroid of the lensed images. With plenty of projects [84-89] monitoring the resulting brightness change of stars, microlensing has been a very important tool in astronomy for searching the unseen components of matter and determining the mass and distance of the lens [90-97]. The weakdeflection picture of microlensing was extended by considering a black hole as the lens [98], which brings the (outer) relativistic images in the strong deflection gravitational lensing into the total magnification and the centroid, turning into the relativistic microlensing. It was shown [98] that, in the case of Sgr A* as the lens, when the lens-source distance is more than $10 \mathrm{pc}$, the relativistic correction due to the outer relativistic images of the Schwarzschild black hole to the microlensing effects are extremely tiny, far beyond the technology in the foreseen future. However, we will show that the correction due to the inner relativistic images of the EinsteinLovelock ultracompact object to the microlensing might be bigger than those due to the outer relativistic images of the Schwarzschild black hole and the Einstein-Lovelock regular black hole by a factor of 100 and demonstrate a potential possibility of testing the Einstein-Lovelock ultracompact object by the relativistic microlensing on a star whose pericenter distance to Sgr A* might reach the level of less than 18 au, just like the S62 star [99].

In the case of the weak deflection gravitational lensing, the closest approach of the photon $r_{0}$ is much larger than $m_{\text {. }}$ so that the metric (3) can be expanded in terms of $m_{\bullet} r^{-1}$. We, therefore, show in Appendix A that the Schwarzschild black hole, the Einstein-Lovelock regular black hole and the Einstein-Lovelock ultracompact object share the same Taylor-expand form of their metrics up to the order of $\mathcal{O}\left(m_{\bullet}^{6} r^{-6}\right)$, making them indistinguishable from the microlensing observables with current technology. These observables of the total magnification and the centroid for the Schwarzschild black hole are already well known [98] and they are listed in Appendix A for selfcompleteness. We will focus on the relativistic microlensing by the Einstein-Lovelock ultracompact object in this section, since the observables for the Einstein-Lovelock regular black hole can be easily recovered from those of the EinsteinLovelock ultracompact object when the contribution from the inner relativistic images is discarded.

Based on Eq. (40), the positions of the positive- and negative-parity outer relativistic images can be found as [98]

$\vartheta_{+n}^{ \pm}= \pm \vartheta_{+n}^{\mathrm{E}}+\frac{u_{m} D_{\mathrm{OS}}}{\bar{a}_{+} D_{\mathrm{LS}} D_{\mathrm{OL}}} e_{+n}\left(\mathcal{B} \mp \vartheta_{+n}^{\mathrm{E}}\right)$,

and, with the help of Eq. (41), their magnification can be obtained as

$\mu_{+n}^{ \pm}=\frac{u_{m}^{2} D_{\mathrm{OS}}}{\bar{a}_{+} \mathcal{B} D_{\mathrm{OL}}^{2} D_{\mathrm{LS}}}\left(1+e_{+n}\right) e_{+n}$.

The total magnification for the outer relativistic images is

$$
\begin{aligned}
\mu_{+, \text {tot }} & =\sum_{n=1}^{\infty}\left(\mu_{+n}^{+}+\mu_{+n}^{-}\right) \\
& =\frac{2 u_{m}^{2} D_{\mathrm{OS}}}{\bar{a}_{+} \mathcal{B} D_{\mathrm{OL}}^{2} D_{\mathrm{LS}}} \sum_{n=1}^{\infty}\left(1+e_{+n}\right) e_{+n} .
\end{aligned}
$$


Likewise, based on Eq. (45), we can have the positions of the positive- and negative-parity inner relativistic images as

$\vartheta_{-n}^{ \pm}= \pm \vartheta_{-n}^{\mathrm{E}}-\frac{u_{m} D_{\mathrm{OS}}}{2 \bar{a}_{-} D_{\mathrm{LS}} D_{\mathrm{OL}}} \frac{e_{-n}\left(\mathcal{B} \mp \vartheta_{-n}^{\mathrm{E}}\right)}{\left(1+e_{-n}\right)^{3 / 2}}$,

and, from Eq. (46), we can find their magnification as

$\mu_{-n}^{ \pm}=\frac{u_{m}^{2} D_{\mathrm{OS}}}{2 \bar{a}_{-} \mathcal{B} D_{\mathrm{OL}}^{2} D_{\mathrm{LS}}} \frac{e_{-n}}{\left(1+e_{-n}\right)^{2}}$.

The total magnification for the inner relativistic images is

$$
\begin{aligned}
\mu_{-, \mathrm{tot}} & =\sum_{n=1}^{\infty}\left(\mu_{-n}^{+}+\mu_{-n}^{-}\right) \\
& =\frac{u_{m}^{2} D_{\mathrm{OS}}}{\bar{a}_{-} \mathcal{B} D_{\mathrm{OL}}^{2} D_{\mathrm{LS}}} \sum_{n=1}^{\infty} \frac{e_{-n}}{\left(1+e_{-n}\right)^{2}} .
\end{aligned}
$$

Therefore, the relativistic total magnification of the outer and inner relativistic images is

$\mu_{\mathrm{tot}}^{\mathrm{s}}=\mu_{+, \mathrm{tot}}+\mu_{-, \mathrm{tot}}=\frac{2 u_{m}^{2} D_{\mathrm{OS}} \mathcal{G}}{\bar{a}_{+} \mathcal{B} D_{\mathrm{OL}}^{2} D_{\mathrm{LS}}}$

where Eq. (33) is used and $\mathcal{G}$ is a summation on all the relativistic images as

$\mathcal{G}=\sum_{n=1}^{\infty}\left[\left(1+e_{+n}\right) e_{+n}+\frac{e_{-n}}{4\left(1+e_{-n}\right)^{2}}\right]$.

For the Einstein-Lovelock ultracompact object, its total magnification for the relativistic microlensing with all the images in the weak and strong deflection gravitational lensing is

$\mu_{\mathrm{tot}}=\mu_{\mathrm{tot}}^{\mathrm{w}}+\mu_{\mathrm{tot}}^{\mathrm{s}}=\mu_{\mathrm{tot}}^{\mathrm{w}}+\mu_{+, \mathrm{tot}}+\mu_{-, \mathrm{tot}}$,

where $\mu_{\text {tot }}^{\mathrm{w}}$ comes from the contribution of the positive- and negative-parity images in the weak deflection gravitational lensing and its expression can be found in Eq. (A.8). It is worth mentioning that, in Eq. (64), its first two terms have been given in the total magnification of the relativistic microlensing for a Schwarzschild black hole considered in Ref. [98], while the third term is unique for an ultracompact object.

As a natural extension of the concept in the microlensing, the centroid of the relativistic microlensing by the EinsteinLovelock ultracompact object is defined as a magnificationweighted summation of all the positive- and negative-parity images in the weak and strong deflection gravitational lensing that is

$$
\begin{aligned}
S_{\mathrm{cen}}= & \frac{1}{\mu_{\mathrm{tot}}}\left[\vartheta^{+} \mu^{+}+\vartheta^{-} \mu^{-}+\sum_{n=1}^{\infty}\left(\vartheta_{+n}^{+} \mu_{+n}^{+}+\vartheta_{+n}^{-} \mu_{+n}^{-}\right)\right. \\
& \left.+\sum_{n=1}^{\infty}\left(\vartheta_{-n}^{+} \mu_{-n}^{+}+\vartheta_{-n}^{-} \mu_{-n}^{-}\right)\right] \\
= & \frac{\mu_{\mathrm{tot}}^{\mathrm{w}}}{\mu_{\mathrm{tot}}} S_{\mathrm{cen}}^{\mathrm{w}}+\frac{\mu_{\mathrm{tot}}^{\mathrm{s}}}{\mu_{\mathrm{tot}}} S_{\mathrm{cen}}^{\mathrm{s}}
\end{aligned}
$$

where the centroid $S_{\mathrm{cen}}^{\mathrm{w}}$ of the lensed images in the weak deflection gravitational lensing can be found in Eq. (A.9) and the relativistic centroid $S_{\text {cen }}^{\text {s }}$ of the outer and inner relativistic images is

$S_{\mathrm{cen}}^{\mathrm{s}}=\frac{\mathcal{B} u_{m} D_{\mathrm{OS}}}{2 \bar{a}_{+} D_{\mathrm{OL}} D_{\mathrm{LS}}} \mathcal{F} \mathcal{G}^{-1}$,

with $\mathcal{F}$ being a summation on all the relativistic images as

$\mathcal{F}=\sum_{n=1}^{\infty}\left[2\left(1+e_{+n}\right) e_{+n}^{2}-\frac{e_{-n}^{2}}{8\left(1+e_{-n}\right)^{7 / 2}}\right]$.

As a comparison with the relativistic centroid considered in Ref. [98], the contribution from the inner relativistic images is unique for an ultracompact object.

Recently, S62 was discovered [100] and confirmed [101] as a star on a 9.9-year orbit around Sgr A* with the pericenter distance $r_{\mathrm{p}, \mathrm{S} 62}=17.8 \mathrm{au}=421.3 \mathrm{~m}_{\bullet}, \mathrm{SgrA} *$. Its velocity at the pericenter can reach about $6.8 \%$ of the speed of light [100], which is $v_{\mathrm{p}, \mathrm{S} 62}=2.053 \times 10^{7} \mathrm{~m} \mathrm{~s}^{-1}$. Although the S62 star would be gravitationally lensed by Sgr A*, the effect is expected to be very weak due to the lack of perfect alignment of them. However, astronomical observations indicate [99] that there might be a population of faint fast-moving stars around Sgr $\mathrm{A}^{*}$ with periods $\lesssim 10 \mathrm{yr}$. We consider a yet-to-be discovered star of this population as a source with $D_{\mathrm{OS}}=r_{\mathrm{p}, \mathrm{S} 62}$. It is assumed to be relativistically microlensed in a nearly collinear configuration by Sgr A*, which is hypothesized as an Einstein-Lovelock ultracompact object. Its angular Einstein ring radius, see Eq. (A.4), is $\vartheta_{\mathrm{E}}=0.208$ mas. We assume that the star will cross the Einstein radius with $v_{\mathrm{E}}=v_{\mathrm{p}, \mathrm{S} 62}$. Its Einstein radius crossing time is defined as

$t_{\mathrm{E}}=\frac{\vartheta_{\mathrm{E}} D_{\mathrm{OL}}}{v_{\mathrm{E}}}=3.51 \mathrm{~h}$,

which characterizes the duration of the relativistic microlensing event. Since $t_{\mathrm{E}}$ is much shorter than the orbital period of the star $\sim 10$ year, its motion during crossing the Einstein ring is able to be approximated to a linear motion with the constant speed $v_{\mathrm{E}}$. Therefore, the angular position of the source can be parameterized as

$\beta=\frac{\mathcal{B}}{\vartheta_{\mathrm{E}}}=\sqrt{\beta_{\text {min }}^{2}+\frac{\left(t-t_{0}\right)^{2}}{t_{\mathrm{E}}^{2}}}$,

where $\beta_{\min }$ is the minimum angular separation between the lens and the source in the unit of $\vartheta_{\mathrm{E}}$ and $t_{0}$ is the time that $\beta$ arrives $\beta_{\min }$. We will examine whether it is possible to distinguish the Einstein-Lovelock ultracompact object from the Schwarzschild black hole and the Einstein-Lovelock regular black hole in this context.

Figure 6 shows color-indexed magnification $\mu_{\text {tot }}^{\mathrm{w}}, \mu_{ \pm \text {, tot }}$ and $\mu_{\text {tot }}$ on the domain of $(\eta, \beta)$ (left column) and their time evolution during crossing the Einstein ring (right column). As 
shown in Appendix A, the total magnification of the images in the weak deflection gravitational lensing $\mu_{\text {tot }}^{\mathrm{W}}$ depends on $\beta$ only and is irrelevant to $\eta$, see Fig. $6 \mathrm{a}$. It increases with $\beta$ and ranges from 1 to about 100 . In the time domain, $\mu_{\text {tot }}^{\mathrm{W}}$ reaches its peak when $\beta$ arrives its minimum, as demonstrated in Fig. 6b. The total magnification of the outer relativistic images in the strong deflection gravitational lensing $\mu_{+}$,tot grows with the increment of $\eta$ roughly, ranging from $\sim 10^{-6.5}$ to $\sim 10^{-3.5}$, see Fig. $6 \mathrm{c}$. In the time domain, we can clearly see from Fig. $6 \mathrm{~d}$ that, for a fixed $\beta_{\min }$, a bigger $\eta$ can make the overall outer relativistic images brighter, i.e. a bigger $\mu_{+, \text {tot }}$. Contrary to $\mu_{+, \text {tot }}$, the total magnification of the inner relativistic images in the strong deflection gravitational lensing $\mu_{- \text {,tot }}$ decreases with the growth of $\eta$ roughly and its value can reach from $\sim 10^{-6}$ to $\sim 10^{-2}$, see Fig. 6e. In the time domain shown in Fig. $6 \mathrm{f}$, when the $\beta_{\text {min }}$ is chosen, a smaller $\eta$ makes the light curve have a bigger peak of $\mu_{-, \text {tot }}$, meaning that the overall inner relativistic images is brighter. Since $\mu_{- \text {,tot }}$ is typically 10 times larger than $\mu_{+}$,tot for the same given $\eta$ and $\beta$, it suggests that the flux of all inner relativistic images in the relativistic microlensing is about 10 times brighter than the one of all outer relativistic images. It also implies that the Einstein-Lovelock ultracompact object might have a brighter peak in the light curve than the Einstein-Lovelock regular black hole, making it easier to detect by photometry, because the much brighter inner relativistic images are a unique feature for ultracompact objects. The total magnification of all images $\mu_{\text {tot }}$ in the relativistic microlensing and its time evolution look very much like those for the images in the weak deflection gravitational lensing due to the faintness of the outer and inner relativistic images, see Fig. 6g, h.

For the total flux observed in practice, we define the following indicators to describe the fractional contributions from different images that are

$\rho_{\mathrm{w}}=\frac{\mu_{\mathrm{tot}}^{\mathrm{W}}}{\mu_{\mathrm{tot}}}, \quad \rho_{+}=\frac{\mu_{+, \mathrm{tot}}}{\mu_{\mathrm{tot}}}$ and $\rho_{-}=\frac{\mu_{-, \mathrm{tot}}}{\mu_{\mathrm{tot}}}$,

which count the contributions from the images in the weak deflection gravitational lensing, the outer and inner relativistic images in the strong deflection gravitational lensing, respectively. From top to bottom, Fig. 7 shows color-indexed $\rho_{\mathrm{W}}$ and $\rho_{ \pm}$on the domain of $(\eta, \beta)$ (left column) and their time evolution during crossing the Einstein ring (right column). As $1-\rho_{\mathrm{w}}$ displayed in Fig. 7a, the fractional contribution from the images in the weak deflection gravitational lensing is very close to 1 , meaning that it dominates the total flux. Although $\mu_{\mathrm{tot}}^{\mathrm{w}}$ is immune to $\eta$, its fractional ratio $\rho_{\mathrm{w}}$ is not by definition, see Eq. (70). It increases with the growth of $\eta$ and $\beta$, and is able to reach $\sim 0.9999$. Figure $7 \mathrm{~b}$ shows some time evolution of $1-\rho_{\mathrm{w}}$ on the time domain. For a given $\beta$, a smaller $\eta$ can result in a bigger peak value of $1-\rho_{\mathrm{W}}$, corresponding to a smaller $\rho_{\mathrm{w}}$. For a fixed $\eta$, a smaller minimum angular separation $\beta_{\text {min }}$ can make the peak value of $1-\rho_{\mathrm{w}}$ bigger. The fractional contribution of the outer relativistic images $\rho_{+}$grows with the increment of $\eta$ and stays at the level of $\sim 10^{-6}$ as shown in Fig. 7c. In the time domain, when $\beta$ is fixed, a bigger $\eta$ allows $\rho_{+}$to have a larger peak value, see Fig. 7d. Unfortunately, since $\rho_{+} \sim 10^{-6}$ is so tiny, it is far beyond the current precision of space-borne photometry about 300 parts per million (ppm). Different from $\rho_{+}$, the fractional contribution of the inner relativistic images $\rho_{-}$ decreases with the growth of $\eta$ as shown in Fig. 7e. It might reach the level of $\sim 10^{-4}$ which is almost 100 times bigger than $\rho_{+}$. In the time domain, after $\beta$ is chosen, a smaller $\eta$ renders $\rho_{-}$with a higher peak, see Fig. 7f. Although $\rho_{-}$is about $100 \mathrm{ppm}$ which is still less than the current photometric resolution by a factor of 3 , it might be possible to distinguish $\rho_{-}$based on its unique dependence of $\eta$ by a dedicated spaceborne equipment in the foreseen future if $\mathrm{Sgr} \mathrm{A} *$ is in its quiescence, implying that the Einstein-Lovelock ultracompact object would be more detectable than the Einstein-Lovelock regular black hole.

Another observable in the relativistic microlensing is the shift of the centroid. In the weak deflection gravitational lensing, it is well known that the centroid shift is [92,95, 102, 103]

$\delta^{\mathrm{w}}=S_{\mathrm{cen}}^{\mathrm{w}}-\mathcal{B}=\frac{\beta}{\beta^{2}+2} \vartheta_{\mathrm{E}}$

and its trajectory is an ellipse with the axis ratio of $\beta_{\min }\left(\beta_{\min }^{2}+2\right)^{-1 / 2}$ when the angular position of the source can be described by Eq. (69). When $\beta=\sqrt{2}$, the centroid shift reaches its maximum as

$\delta_{\max }^{\mathrm{w}}=\frac{\sqrt{2}}{4} \vartheta_{\mathrm{E}}$

If the images in the weak deflection gravitational lensing and the outer relativistic images are considered only, the centroid shift reads

$\delta_{+}=\left.S_{\mathrm{cen}}\right|_{\mu_{-n}^{ \pm}=0}-\mathcal{B}$,

where the contributions of the inner relativistic images are set to be vanished. For the Einstein-Lovelock ultracompact object, its centroid shift is

$\delta_{-}=S_{\mathrm{cen}}-\mathcal{B}$.

In order to show the contributions of the images in the weak deflection gravitational lensing, the outer and inner relativistic images in the strong deflection gravitational lensing in the centroid shift respectively, we define following indicators as

$\delta_{\mathrm{w},+}=\delta^{\mathrm{w}}-\delta_{+}$,
$\delta_{\mathrm{w},-}=\delta^{\mathrm{w}}-\delta_{-}$,
$\delta_{+,-}=\delta_{+}-\delta_{-}$. 
Fig. 6 Left column: color-indexed magnification $\mu_{\text {tot }}^{\mathrm{w}}, \mu_{ \pm \text {, tot }}$ and $\mu_{\text {tot }}$ on the domain of $(\eta, \beta)$. Right column: their time evolution during crossing the Einstein ring (a)

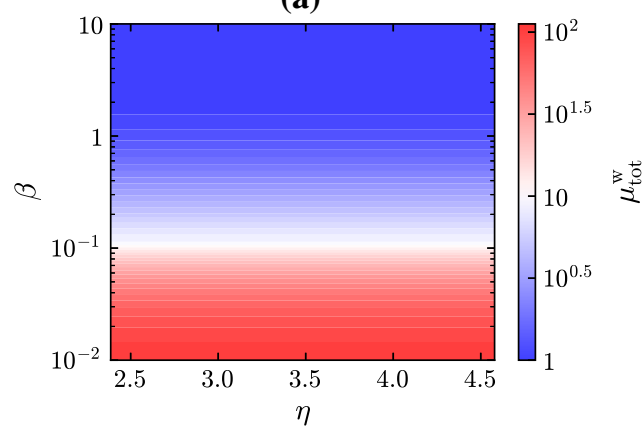

(c)

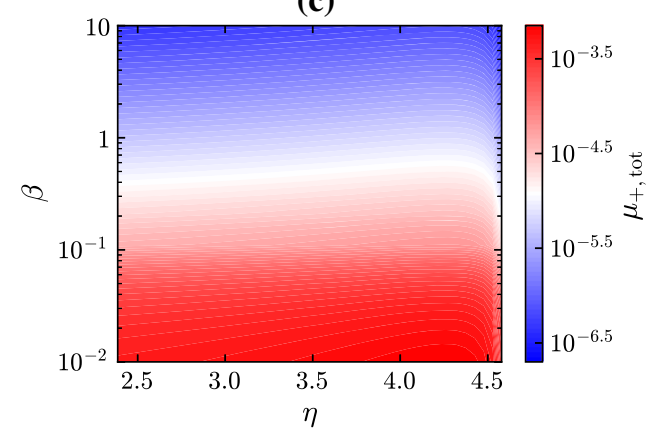

(e)
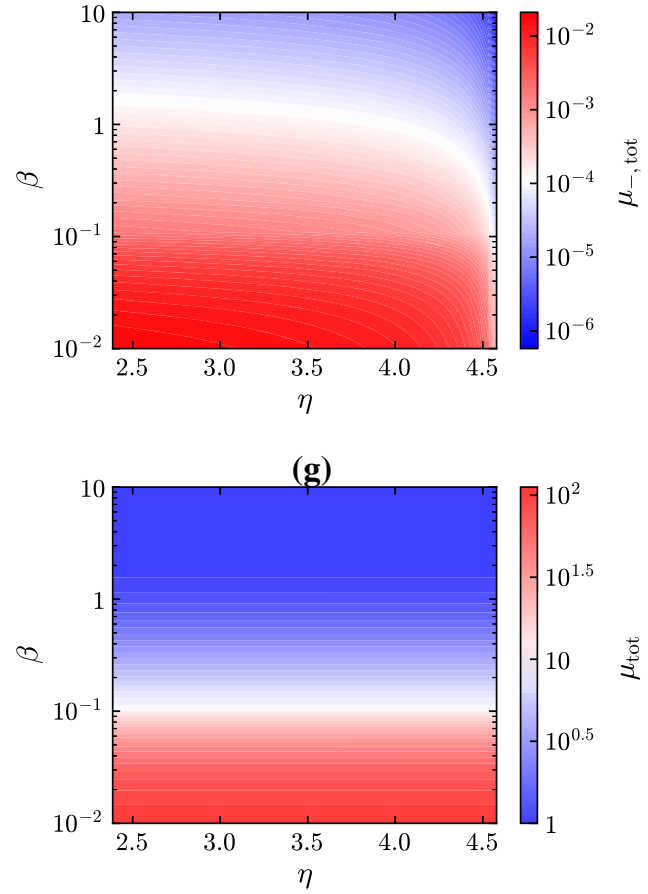

(b)

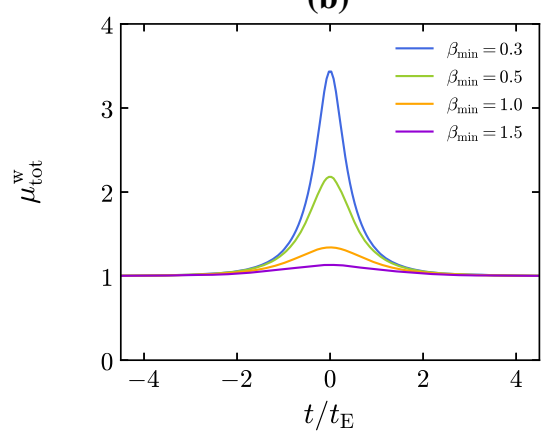

(d)

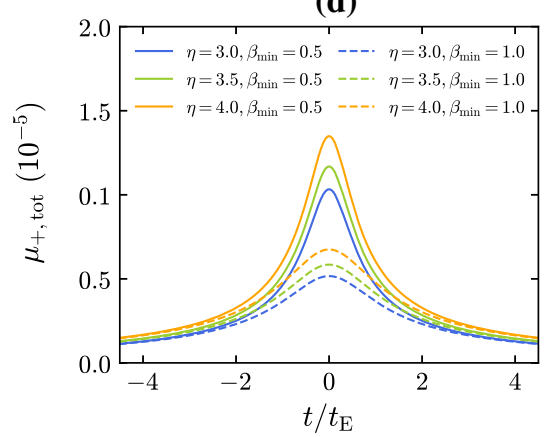

(f)

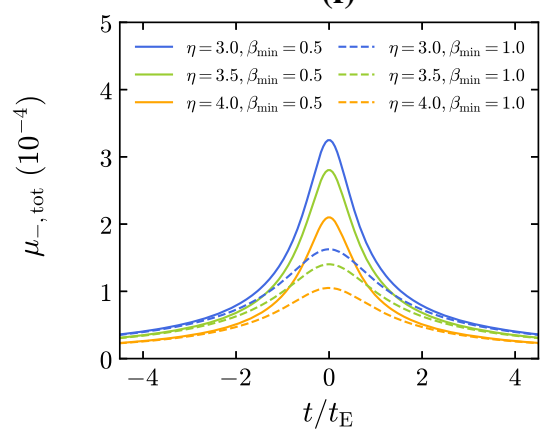

(h)

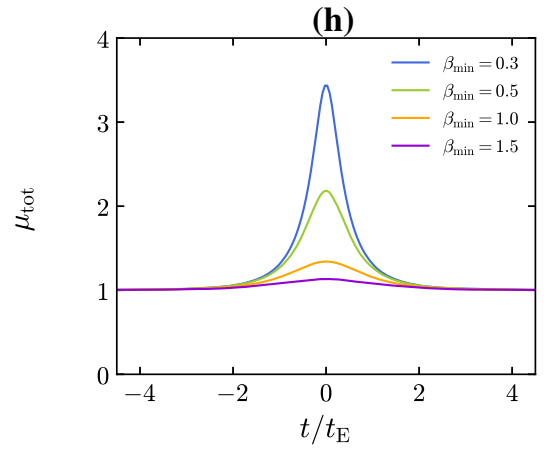

Even before specific calculation, we can expect that, due to the existence of the outer and inner relativistic images, the centroid shift $\delta_{ \pm}$would move inward and become closer to the lens than $\delta^{\mathrm{w}}$, i.e., $\delta^{\mathrm{w}}>\delta_{+}>\delta_{-}$.

For the S62-like source we consider, the maximum centroid shift in the weak deflection gravitational lensing is $\delta_{\max }^{\mathrm{w}}=73.5 \mu$ as which is reachable by current astrometric accuracy of GRAVITY as 10-20 $\mu$ as [28,104]. From top to bottom, Fig. 8 shows color-indexed $\delta_{\mathrm{w}, \pm}$ and $\delta_{+,-}$on the domain of $(\eta, \beta)$ (left column) and their time evolution during crossing the Einstein ring (right column). As displayed in Fig. 8a, the deviation of centroid shift caused by the outer relativistic images $\delta_{\mathrm{w},+}$ increases with $\eta$ and its biggest value is about $1.6 \times 10^{-3} \mu$ as which is far beyond the reach of current technology. Figure $8 \mathrm{~b}$ shows some time evolution of $\delta_{\mathrm{w},+}$ on the time domain. The difference between the light curves with the same $\eta$ but different $\beta$ becomes distinct only when $-2 t_{\mathrm{E}}<t<2 t_{\mathrm{E}}$. For a given $\beta$, a bigger $\eta$ can give a 
Fig. 7 Left column: the color-indexed fractional contributions $\rho_{\mathrm{w}}$ and $\rho_{ \pm}$on the domain of $(\eta, \beta)$. Right column: their time evolution during crossing the Einstein ring (a)

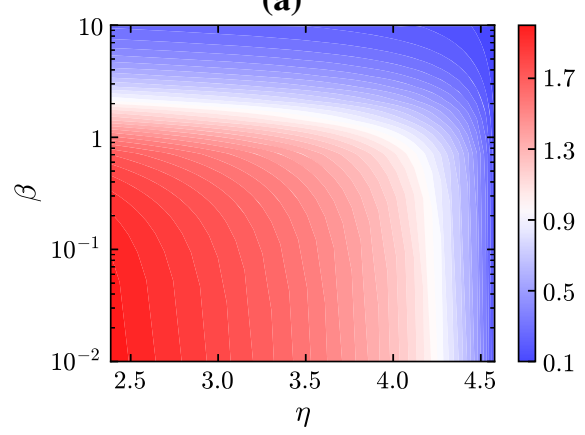

(c)

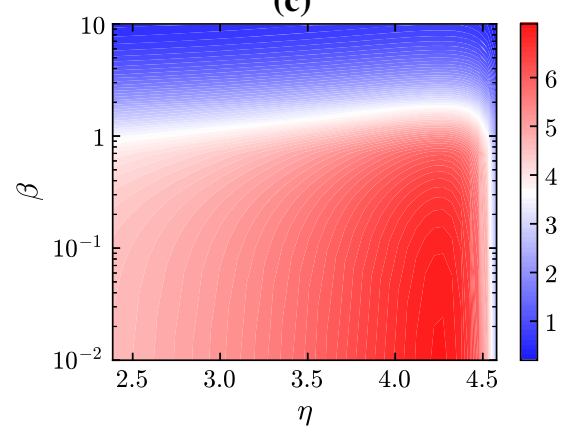

(e)

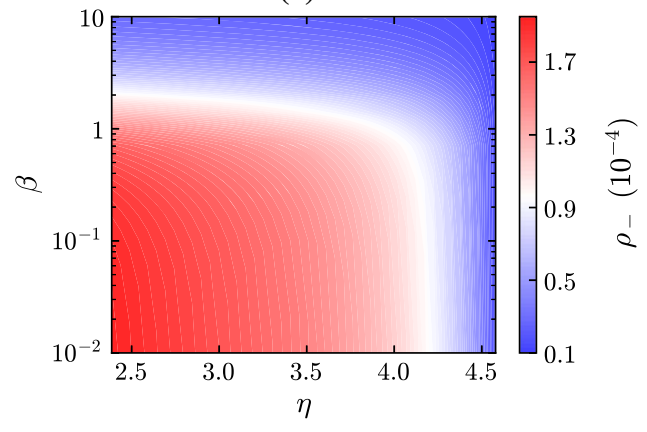

(b)

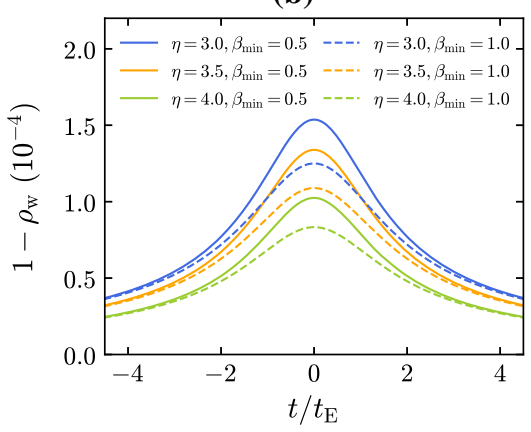

(d)

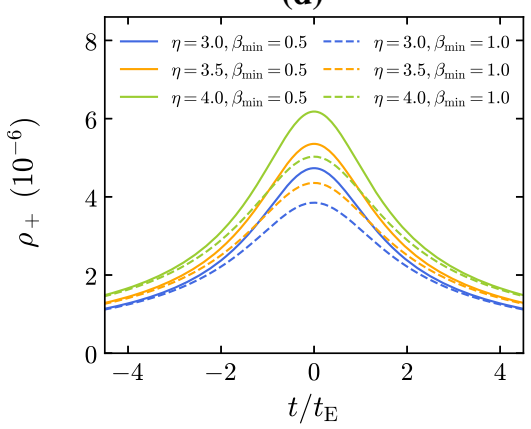

(f)

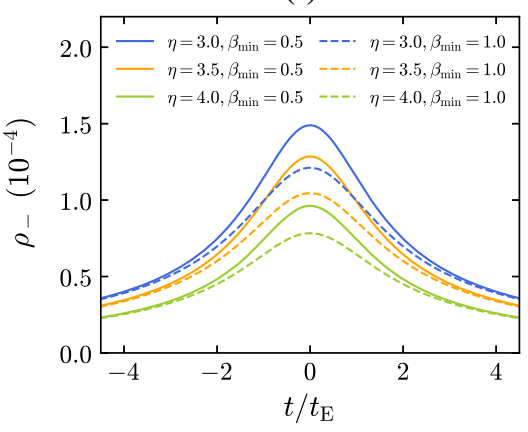

bigger $\delta_{\mathrm{w},+}$. For a fixed $\eta$, a bigger minimum angular separation $\beta_{\text {min }}$ can make $\delta_{\mathrm{w},+}$ larger. The deviation of centroid shift due to both outer and inner relativistic images $\delta_{\mathrm{w},-}$ decreases with $\eta$ and can reach the level of $\sim 10^{-2} \mu$ as as shown in Fig. 8c. In the time domain, when $\beta$ is fixed, a bigger $\eta$ renders $\delta_{\mathrm{w},-}$ smaller, which is contrary to the trend of $\delta_{\mathrm{w},+}$, see Fig. 8d. Although $\delta_{\mathrm{w},-}$ is about 100 times bigger than $\delta_{\mathrm{w},+}$, it is still too tiny to measure. The deviation of centroid shift solely contributed by the inner relativistic images $\delta_{+,-}$and its time evolution as shown in Fig. 8e, f are very similar to those of $\delta_{\mathrm{w},-}$ because the outer relativistic images are much fainter than the inner relativistic images. Therefore, it is infeasible to detect $\delta_{\mathrm{w}, \pm}$ and $\delta_{+,-}$by current astrometry.

In a summary, when considering a S62-like star relativistically microlensed by $\mathrm{Sgr} \mathrm{A}^{*}$, we find that (1) it might be possible to distinguish the Einstein-Lovelock ultracompact object from the Einstein-Lovelock regular black hole by measuring the effects of the inner relativistic images on the total flux and its variation with time during a quiet state of
Sgr A* in the not-so-far future; and (2) it is impossible to distinguish any contribution from either outer or inner relativistic images by measuring the centroid shift of the relativistic microlensing by the Einstein-Lovelock regular black hole or ultracompact object.

\section{Conclusions and discussion}

We investigate the strong deflection gravitational lensing by the Einstein-Lovelock ultracompact object predicted by a special class of the four-dimensional regularized EinsteinLovelock gravity. Its unique lensing observables are the inner relativistic images which are absent for the EinsteinLovelock regular black hole given by the same theory. For the direct images of Sgr A* and M87*, we study the angular separations and the brightness differences among the outermost relativistic image, the photon sphere and the innermost relativistic image for the Einstein-Lovelock ultracompact object 
Fig. 8 Left column: the color-indexed centroid shift $\delta_{\mathrm{w}, \pm}$ and $\delta_{+,-}$on the domain of $(\eta, \beta)$. Right column: their time evolution during crossing the Einstein ring (a)

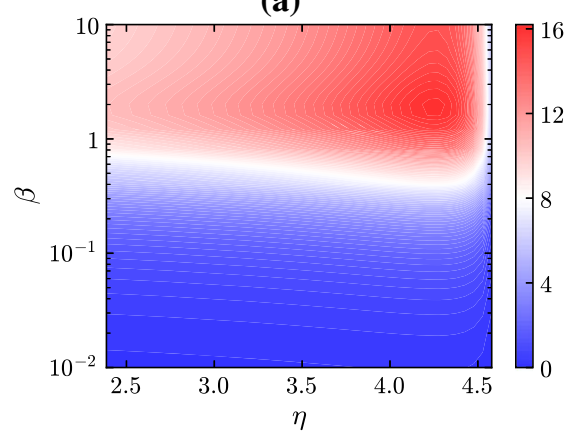

(c)

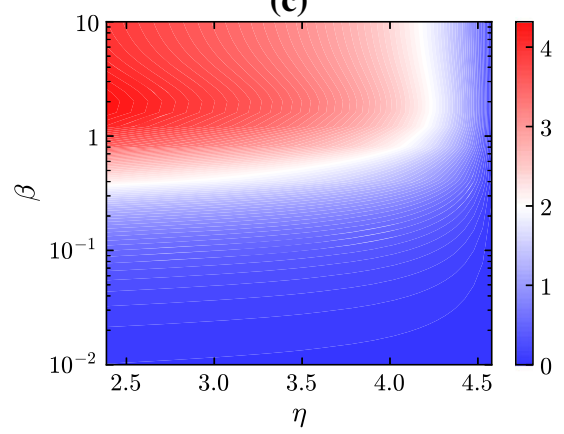

(e)

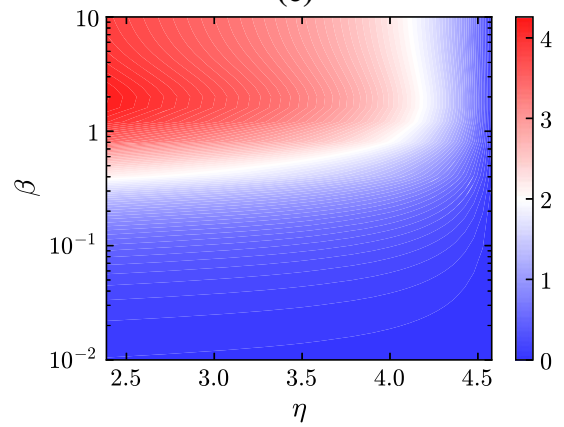

(b)

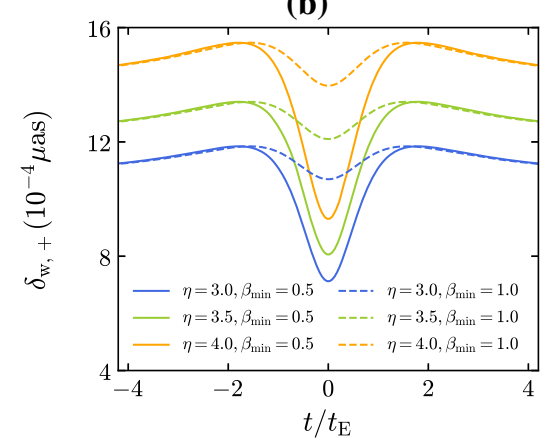

(d)

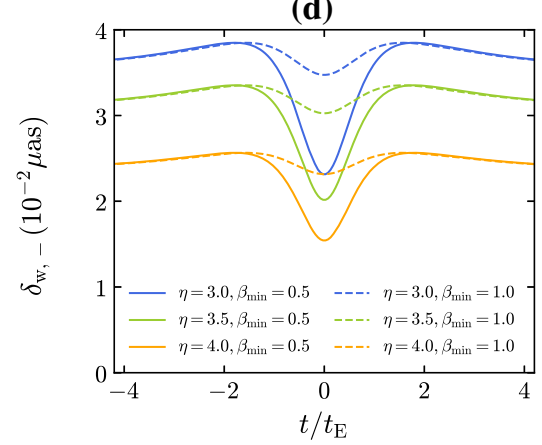

(f)

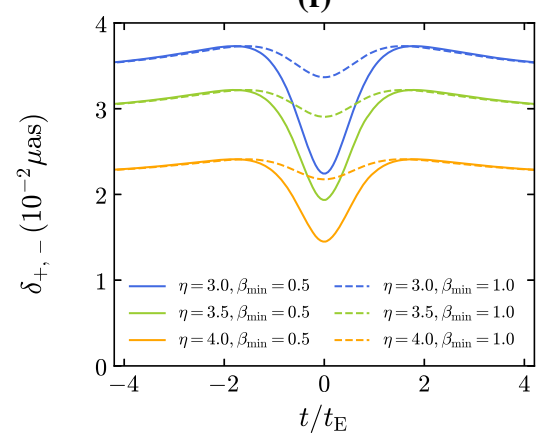

and regular black hole (if applicable). We find that although the angular separation and the brightness of the innermost relativistic image of the Einstein-Lovelock ultracompact object are more significant than those of the outermost relativistic one, it is impossible to tell difference from the Schwarzschild black hole, the Einstein-Lovelock regular black hole and ultracompact object based on the measured size of M87*'s shadow by EHT. Furthermore, considering a S62-like star relativistically microlensed by Sgr A*, we find that although the centroid shift of the relativistic microlensing is far from reachable for the current astrometry, it might be possible to distinguish the Einstein-Lovelock ultracompact object from the Einstein-Lovelock regular black hole by measuring the effects of the inner relativistic images on the total flux and its variation with time in the not-so-far future.

In this work, we do not take the rotation of the EinsteinLovelock ultracompact object into account. A non-rotating M87* is disfavored by the observation of EHT [7]. However, the metric of a spinning Einstein-Lovelock ultracom- pact object is still unknown for now and, therefore, its gravitational lensing signatures cannot be found self-consistently. Even though, we could expect that the spin might shift and distort the caustic, like in other scenarios [105-122]. In order to interpret the observed size and shape of M87* in the direct image by EHT, one has to heavily rely on computationally expensive simulations of general relativistic magnetohydrodynamics of plasma around the Kerr black hole [11], in which plenty of untested assumptions about accretion flow and emission physics are made [123]. Any deviation in the spacetime raised from the Einstein-Lovelock theory might not be distinguished from the breakdown of these astrophysical assumptions. The photon ring [124] around M87* might offer a vigorous test, but it needs very challenging spacebased interferometry [125-127]. Therefore, we cannot constrain the Einstein-Lovelock ultracompact object and regular black hole based on the direct images of Sgr A* and M87* in a self-consistent way at least in the current stage. This situation pushes us to find that the relativistic microlensing 
on a S62-like by the quiescent Sgr A* might be a promising way to distinguish the Einstein-Lovelock ultracompact object. Nevertheless, separating its signals from astrophysical noises, such as flares of Sgr A* and brightness variation due to tidal interaction with Sgr A*, would be far from easy. In view of these difficulties, it would be also necessary to know more tell-tale signatures about the Einstein-Lovelock ultracompact object, for instance the precessing [128-135] and periodic [136-147] orbits around it.

Acknowledgements This work is funded by the National Natural Science Foundation of China (Grant Nos. 11573015 and 11833004).

Data Availability Statement This manuscript has no associated data or the data will not be deposited. [Authors' comment: This paper is a theoretical work and all of the data are adopted by the related references.]

Open Access This article is licensed under a Creative Commons Attribution 4.0 International License, which permits use, sharing, adaptation, distribution and reproduction in any medium or format, as long as you give appropriate credit to the original author(s) and the source, provide a link to the Creative Commons licence, and indicate if changes were made. The images or other third party material in this article are included in the article's Creative Commons licence, unless indicated otherwise in a credit line to the material. If material is not included in the article's Creative Commons licence and your intended use is not permitted by statutory regulation or exceeds the permitted use, you will need to obtain permission directly from the copyright holder. To view a copy of this licence, visit http://creativecomm ons.org/licenses/by/4.0/.

Funded by SCOAP ${ }^{3}$.

\section{Appendix A: Weak deflection gravitational lensing}

In the weak deflection gravitational lensing, the closest approach of the photon $r_{0}$ is much larger than $m_{\bullet}$ so that the metric (3) can be expanded in terms of $m_{\bullet} r^{-1}$ as

$$
\begin{aligned}
A(r)= & 1-2 \frac{m_{\bullet}}{r}+\frac{4}{3} \eta_{\bullet}^{2} \frac{m_{\bullet}^{7}}{r^{7}}+\mathcal{O}\left(\frac{m_{\bullet}^{13}}{r^{13}}\right), \\
B(r)= & 1+2 \frac{m_{\bullet}}{r}+4 \frac{m_{\bullet}^{2}}{r^{2}}+8 \frac{m_{\bullet}^{3}}{r^{3}}+16 \frac{m_{\bullet}^{4}}{r^{4}} \\
& +32 \frac{m_{\bullet}^{5}}{r^{5}}-\frac{4}{3} \eta_{\bullet}^{2} \frac{m_{\bullet}^{7}}{r^{7}}+\mathcal{O}\left(\frac{m_{\bullet}^{8}}{r^{8}}\right) .
\end{aligned}
$$

We find that $A(r)$ and $B(r)$ deviate from those of the Schwarzschild black hole starting at the orders of $\mathcal{O}\left(m^{7} r^{-7}\right)$ and $\mathcal{O}\left(m_{\bullet}^{6} r^{-6}\right)$, respectively. Nevertheless, these tiny deviations are far beyond the reach of current technology so that it is impossible to distinguish the Schwarzschild black hole, the Einstein-Lovelock regular black hole and the EinsteinLovelock ultracompact object through measuring observables in the weak deflection gravitational lensing. For selfcompleteness, we will list the lensing observables in the weak deflection scenario for the spacetime (A.1) and (A.2) based on the well-known ones for the Schwarzschild spacetime.
For the convenience, we also define the following dimensionless variables

$\beta=\frac{\mathcal{B}}{\vartheta_{\mathrm{E}}}$ and $\theta=\frac{\vartheta}{\vartheta_{\mathrm{E}}}$,

where $\vartheta_{\mathrm{E}}$ is the angular Einstein ring radius

$\vartheta_{\mathrm{E}}=\sqrt{\frac{4 m_{\bullet} D_{\mathrm{LS}}}{D_{\mathrm{OL}} D_{\mathrm{OS}}}}$.

For the spacetime (A.1) and (A.2), the positions of positiveand negative-parity images $\theta^{ \pm}$can be obtained as [98]

$\theta^{ \pm}=\frac{1}{2}(\beta \pm \eta)$,

where

$\eta=\sqrt{4+\beta^{2}}$,

and their magnification $\mu^{ \pm}$can be found as [98]

$\mu^{ \pm}= \pm \frac{1}{2}+\frac{\beta^{2}+2}{2 \beta \eta}$

When the positive- and negative-parity images are unresolved, the total magnification and the centroid of these two images will be the observables. The total magnification is [98]

$\mu_{\mathrm{tot}}^{\mathrm{w}}=\mu^{+}+\mu^{-}=\frac{\beta^{2}+2}{\beta \eta}$.

As a magnification-weighted summation of the positive- and negative-parity images, the centroid is [98]

$S_{\mathrm{cen}}^{\mathrm{w}}=\frac{\vartheta^{+} \mu^{+}+\vartheta^{-} \mu^{-}}{\mu^{+}+\mu^{-}}=\mathcal{B} \frac{\beta^{2}+3}{\beta^{2}+2}$.

\section{References}

1. B.P. Abbott et al. (LIGO Scientific and Virgo Collaborations), Phys. Rev. Lett. 116(6), 061102 (2016). https://doi.org/10.1103/ PhysRevLett.116.061102

2. B.P. Abbott et al. (LIGO Scientific and Virgo Collaborations), Phys. Rev. X 6(4), 041015 (2016). https://doi.org/10.1103/ PhysRevX.6.041015

3. B.P. Abbott et al. (LIGO Scientific and Virgo Collaborations), Phys. Rev. Lett. 116(24), 241103 (2016). https://doi.org/10.1103/ PhysRevLett.116.241103

4. B.P. Abbott et al. (LIGO Scientific and Virgo Collaborations), Phys. Rev. Lett. 118(22), 221101 (2017). https://doi.org/10.1103/ PhysRevLett.118.221101

5. B.P. Abbott et al. (LIGO Scientific and Virgo Collaborations), Astrophys. J. Lett. 851, L35 (2017). https://doi.org/10.3847/ 2041-8213/aa9f0c

6. B.P. Abbott et al. (LIGO Scientific and Virgo Collaborations), Phys. Rev. Lett. 119(14), 141101 (2017). https://doi.org/10.1103/ PhysRevLett.119.141101

7. K. Akiyama et al. (Event Horizon Telescope Collaboration), Astrophys. J. Lett. 875, L1 (2019). https://doi.org/10.3847/ 2041-8213/ab0ec7 
8. K. Akiyama et al. (Event Horizon Telescope Collaboration), Astrophys. J. Lett. 875, L2 (2019). https://doi.org/10.3847/ 2041-8213/ab0c96

9. K. Akiyama et al. (Event Horizon Telescope Collaboration), Astrophys. J. Lett. 875, L3 (2019). https://doi.org/10.3847/ 2041-8213/ab0c57

10. K. Akiyama et al. (Event Horizon Telescope Collaboration), Astrophys. J. Lett. 875, L4 (2019). https://doi.org/10.3847/ 2041-8213/ab0e85

11. K. Akiyama et al. (Event Horizon Telescope Collaboration), Astrophys. J. Lett. 875, L5 (2019). https://doi.org/10.3847/ 2041-8213/ab0f43

12. K. Akiyama et al. (Event Horizon Telescope Collaboration), Astrophys. J. Lett. 875, L6 (2019). https://doi.org/10.3847/ 2041-8213/ab1141

13. R. Carballo-Rubio, F. Di Filippo, S. Liberati, M. Visser, Phys. Rev. D 98(12), 124009 (2018). https://doi.org/10.1103/PhysRevD.98. 124009

14. V. Cardoso, P. Pani, Nat. Astron. 1, 586 (2017). https://doi.org/ 10.1038/s41550-017-0225-y

15. V. Cardoso, P. Pani, Living Rev. Relativ. 22(1), 4 (2019). https:// doi.org/10.1007/s41114-019-0020-4

16. R.A. Konoplya, A. Zhidenko, Phys. Rev. D 101(8), 084038 (2020). https://doi.org/10.1103/PhysRevD.101.084038

17. A. Casalino, A. Colléaux, M. Rinaldi, S. Vicentini, Phys. Dark Univ. 31, 100770 (2021). https://doi.org/10.1016/j.dark.2020. 100770

18. D. Lovelock, J. Math. Phys. 12, 498 (1971). https://doi.org/10. $1063 / 1.1665613$

19. R.A. Konoplya, T.D. Pappas, Z. Stuchlík, Phys. Rev. D 102(8), 084043 (2020). https://doi.org/10.1103/PhysRevD.102.084043

20. R.A. Konoplya, A. Zhidenko, Phys. Dark Univ. 30, 100697 (2020). https://doi.org/10.1016/j.dark.2020.100697

21. R.A. Konoplya, A. Zhidenko, Phys. Lett. B 807, 135607 (2020). https://doi.org/10.1016/j.physletb.2020.135607

22. C. Gao, S. Yu, J. Qiu, Phys. Dark Univ. 31, 100754 (2021). https:// doi.org/10.1016/j.dark.2020.100754

23. V. Cardoso, E. Franzin, P. Pani, Phys. Rev. Lett. 116(17), 171101 (2016). https://doi.org/10.1103/PhysRevLett.116.171101

24. V. Cardoso, S. Hopper, C.F.B. Macedo, C. Palenzuela, P. Pani, Phys. Rev. D 94(8), 084031 (2016). https://doi.org/10.1103/ PhysRevD.94.084031

25. H. Nakano, N. Sago, H. Tagoshi, T. Tanaka, Prog. Theor. Exp. Phys. 2017(7), 071E01 (2017). https://doi.org/10.1093/ptep/ ptx093

26. A. Urbano, H. Veermäe, J. Cosmol. Astropart. Phys. 2019(4), 011 (2019). https://doi.org/10.1088/1475-7516/2019/04/011

27. R.A. Konoplya, Z. Stuchlík, A. Zhidenko, Phys. Rev. D 99(2), 024007 (2019). https://doi.org/10.1103/PhysRevD.99.024007

28. GRAVITY Collaboration, Astron. Astrophys. 602, A94 (2017). https://doi.org/10.1051/0004-6361/201730838

29. C. Darwin, Proc. R. Soc. A 249, 180 (1959). https://doi.org/10. 1098/rspa.1959.0015

30. V. Bozza, Gen. Relativ. Gravit. 42, 2269 (2010). https://doi.org/ 10.1007/s10714-010-0988-2

31. P.V.P. Cunha, C.A.R. Herdeiro, Gen. Relativ. Gravit. 50, 42 (2018). https://doi.org/10.1007/s10714-018-2361-9

32. K.S. Virbhadra, D. Narasimha, S.M. Chitre, Astron. Astrophys. 337, 1 (1998)

33. K.S. Virbhadra, G.F.R. Ellis, Phys. Rev. D 62(8), 084003 (2000)

34. V. Bozza, Phys. Rev. D 66(10), 103001 (2002). https://doi.org/ 10.1103/PhysRevD.66.103001

35. E.F. Eiroa, G.E. Romero, D.F. Torres, Phys. Rev. D 66(2), 024010 (2002). https://doi.org/10.1103/PhysRevD.66.024010

36. V. Bozza, Phys. Rev. D 67(10), 103006 (2003). https://doi.org/ 10.1103/PhysRevD.67.103006
37. S.E. Vázquez, E.P. Esteban, Nuovo Cimento B Ser. 119, 489 (2004). https://doi.org/10.1393/ncb/i2004-10121-y

38. N. Tsukamoto, Phys. Rev. D 95(6), 064035 (2017). https://doi. org/10.1103/PhysRevD.95.064035

39. T. Hsieh, D.S. Lee, C.Y. Lin, Phys. Rev. D 103(10), 104063 (2021). https://doi.org/10.1103/PhysRevD.103.104063

40. A.Y. Bin-Nun, Phys. Rev. D 81(12), 123011 (2010). https://doi. org/10.1103/PhysRevD.81.123011

41. S.S. Zhao, Y. Xie, J. Cosmol. Astropart. Phys. 07, 007 (2016). https://doi.org/10.1088/1475-7516/2016/07/007

42. X. Lu, F.W. Yang, Y. Xie, Eur. Phys. J. C 76, 357 (2016). https:// doi.org/10.1140/epjc/s10052-016-4218-2

43. S.S. Zhao, Y. Xie, Eur. Phys. J. C 77, 272 (2017). https://doi.org/ 10.1140/epjc/s10052-017-4850-5

44. S.S. Zhao, Y. Xie, Phys. Lett. B 774, 357 (2017). https://doi.org/ 10.1016/j.physletb.2017.09.090

45. S. Chakraborty, S. SenGupta, J. Cosmol. Astropart. Phys. 7, 045 (2017). https://doi.org/10.1088/1475-7516/2017/07/045

46. F.Y. Liu, Y.F. Mai, W.Y. Wu, Y. Xie, Phys. Lett. B 795, 475 (2019). https://doi.org/10.1016/j.physletb.2019.06.052

47. C.Y. Wang, Y.F. Shen, Y. Xie, J. Cosmol. Astropart. Phys. 04, 022 (2019). https://doi.org/10.1088/1475-7516/2019/04/022

48. X. Lu, Y. Xie, Eur. Phys. J. C 79(12), 1016 (2019). https://doi. org/10.1140/epjc/s10052-019-7537-2

49. X. Lu, Y. Xie, Eur. Phys. J. C 80(7), 625 (2020). https://doi.org/ 10.1140/epjc/s10052-020-8205-2

50. Y.X. Gao, Y. Xie, Phys. Rev. D 103, 043008 (2021). https://doi. org/10.1103/PhysRevD.103.043008

51. X. Lu, Y. Xie, Eur. Phys. J. C 81(7), 627 (2021). https://doi.org/ 10.1140/epjc/s10052-021-09440-x

52. P.V.P. Cunha, E. Berti, C.A.R. Herdeiro, Phys. Rev. Lett. 119(25), 251102 (2017). https://doi.org/10.1103/PhysRevLett. 119.251102

53. S. Hod, Phys. Lett. B 776, 1 (2018). https://doi.org/10.1016/j. physletb.2017.11.021

54. P.V.P. Cunha, C.A.R. Herdeiro, Phys. Rev. Lett. 124(18), 181101 (2020). https://doi.org/10.1103/PhysRevLett.124.181101

55. P.V.P. Cunha, J. Grover, C. Herdeiro, E. Radu, H. Rúnarsson, A. Wittig, Phys. Rev. D 94(10), 104023 (2016). https://doi.org/10. 1103/PhysRevD.94.104023

56. H.C.D.L. Junior, P.V.P. Cunha, C.A.R. Herdeiro, L.C.B. Crispino, Phys. Rev. D 104(4), 044018 (2021). https://doi.org/10.1103/ PhysRevD.104.044018

57. R. Shaikh, P. Banerjee, S. Paul, T. Sarkar, Phys. Rev. D 99(10), 104040 (2019). https://doi.org/10.1103/PhysRevD.99.104040

58. J.G. Cramer, R.L. Forward, M.S. Morris, M. Visser, G. Benford, G.A. Landis, Phys. Rev. D 51(6), 3117 (1995). https://doi.org/10. 1103/PhysRevD.51.3117

59. K.K. Nandi, Y.Z. Zhang, A.V. Zakharov, Phys. Rev. D 74(2), 024020 (2006). https://doi.org/10.1103/PhysRevD.74.024020

60. C. Bambi, Phys. Rev. D 87(10), 107501 (2013). https://doi.org/ 10.1103/PhysRevD.87.107501

61. K.K. Nandi, R.N. Izmailov, E.R. Zhdanov, A. Bhattacharya, J. Cosmol. Astropart. Phys. 2018(7), 027 (2018). https://doi.org/10. 1088/1475-7516/2018/07/027

62. K. Jusufi, N. Sarkar, F. Rahaman, A. Banerjee, S. Hansraj, Eur. Phys. J. C 78(4), 349 (2018). https://doi.org/10.1140/epjc/ s10052-018-5823-z

63. K. Jusufi, A. Övgün, Phys. Rev. D 97(2), 024042 (2018). https:// doi.org/10.1103/PhysRevD.97.024042

64. R. Shaikh, Phys. Rev. D 98(2), 024044 (2018). https://doi.org/10. 1103/PhysRevD.98.024044

65. R. Shaikh, P. Banerjee, S. Paul, T. Sarkar, Phys. Lett. B 789, 270 (2019). https://doi.org/10.1016/j.physletb.2018.12.030 
66. R. Shaikh, P. Banerjee, S. Paul, T. Sarkar, J. Cosmol. Astropart. Phys. 2019(7), 028 (2019). https://doi.org/10.1088/1475-7516/ 2019/07/028

67. M. Amir, K. Jusufi, A. Banerjee, S. Hansraj, Class. Quantum Gravity 36(21), 215007 (2019). https://doi.org/10.1088/1361-6382/ ab42be

68. S. Sahu, M. Patil, D. Narasimha, P.S. Joshi, Phys. Rev. D 86(6), 063010 (2012). https://doi.org/10.1103/PhysRevD.86.063010

69. R. Shaikh, P. Kocherlakota, R. Narayan, P.S. Joshi, Mon. Not. R. Astron. Soc. 482(1), 52 (2019). https://doi.org/10.1093/mnras/ sty 2624

70. T. Kubo, N. Sakai, Phys. Rev. D 93(8), 084051 (2016). https:// doi.org/10.1103/PhysRevD.93.084051

71. P.V.P. Cunha, J.A. Font, C. Herdeiro, E. Radu, N. Sanchis-Gual, M. Zilhão, Phys. Rev. D 96(10), 104040 (2017). https://doi.org/ 10.1103/PhysRevD.96.104040

72. M. Patil, P. Mishra, D. Narasimha, Phys. Rev. D 95(2), 024026 (2017). https://doi.org/10.1103/PhysRevD.95.024026

73. X.Y. Zhu, Y. Xie, Eur. Phys. J. C 80(5), 444 (2020). https://doi. org/10.1140/epjc/s10052-020-8021-8

74. G. Kofinas, R. Olea, J. High Energy Phys. 2007(11), 069 (2007). https://doi.org/10.1088/1126-6708/2007/11/069

75. R.A. Konoplya, A. Zhidenko, J. Cosmol. Astropart. Phys. 2017(5), 050 (2017). https://doi.org/10.1088/1475-7516/2017/ $05 / 050$

76. G. Kunstatter, H. Maeda, T. Taves, Class. Quantum Gravity 33(10), 105005 (2016). https://doi.org/10.1088/0264-9381/33/ $10 / 105005$

77. J.L. Synge, Mon. Not. R. Astron. Soc. 131, 463 (1966). https:// doi.org/10.1093/mnras/131.3.463

78. Event Horizon Telescope. Collaboration, Phys. Rev. Lett. 125, 141104 (2020). https://doi.org/10.1103/PhysRevLett.125. 141104

79. D. Psaltis, F. Özel, C.K. Chan, D.P. Marrone, Astrophys. J. 814, 115 (2015). https://doi.org/10.1088/0004-637X/814/2/115

80. S. Weinberg, Gravitation and cosmology: principles and applications of the general theory of relativity (Wiley, New York, 1972)

81. S. Refsdal, Mon. Not. R. Astron. Soc. 128, 295 (1964). https:// doi.org/10.1093/mnras/128.4.295

82. V. Bozza, S. Capozziello, G. Iovane, G. Scarpetta, Gen. Relativ. Gravit. 33, 1535 (2001). https://doi.org/10.1023/A: 1012292927358

83. S. Gillessen, P.M. Plewa, F. Eisenhauer, R. Sari et al., Astrophys. J. 837, 30 (2017). https://doi.org/10.3847/1538-4357/aa5c41

84. C. Alcock, C.W. Akerlof, R.A. Allsman, T.S. Axelrod, D.P. Bennett, S. Chan, K.H. Cook, K.C. Freeman, K. Griest, S.L. Marshall, H.S. Park, S. Perlmutter, B.A. Peterson, M.R. Pratt, P.J. Quinn, A.W. Rodgers, C.W. Stubbs, W. Sutherland, Nature 365, 621 (1993). https://doi.org/10.1038/365621a0

85. A. Udalski, M. Szymanski, J. Kaluzny, M. Kubiak, W. Krzeminski, M. Mateo, G.W. Preston, B. Paczynski, Acta Astron. 43, 289 (1993)

86. E. Aubourg, P. Bareyre, S. Bréhin, M. Gros, M. Lachièze-Rey, B. Laurent, E. Lesquoy, C. Magneville, A. Milsztajn, L. Moscoso, F. Queinnec, J. Rich, M. Spiro, L. Vigroux, S. Zylberajch, R. Ansari, F. Cavalier, M. Moniez, J.P. Beaulieu, R. Ferlet, P. Grison, A. Vidal-Madjar, J. Guibert, O. Moreau, F. Tajahmady, E. Maurice, L. Prévôt, C. Gry, Nature 365, 623 (1993). https://doi.org/10.1038/ $365623 \mathrm{a} 0$

87. C. Alard, J. Guibert, O. Bienayme, D. Valls-Gabaud, A.C. Robin, A. Terzan, E. Bertin, Messenger 80, 31 (1995)

88. I.A. Bond, F. Abe, R.J. Dodd, J.B. Hearnshaw, M. Honda, J. Jugaku, P.M. Kilmartin, A. Marles, K. Masuda, Y. Matsubara, Y. Muraki, T. Nakamura, G. Nankivell, S. Noda, C. Noguchi, K. Ohnishi, N.J. Rattenbury, M. Reid, T. Saito, H. Sato, M. Sekiguchi, J. Skuljan, D.J. Sullivan, T. Sumi, M. Takeuti, Y.
Watase, S. Wilkinson, R. Yamada, T. Yanagisawa, P.C.M. Yock, Mon. Not. R. Astron. Soc. 327, 868 (2001). https://doi.org/10. 1046/j.1365-8711.2001.04776.x

89. M.G. Navarro, D. Minniti, R. Contreras Ramos, Astrophys. J. Lett. 851, L13 (2017). https://doi.org/10.3847/2041-8213/aa9b29

90. B. Paczynski, Acta Astron. 45, 345 (1995)

91. B. Paczynski, Acta Astron. 45, 349 (1995)

92. E. Høg, I.D. Novikov, A.G. Polnarev, Astron. Astrophys. 294, 287 (1995)

93. B. Paczynski, Ann. Rev. Astron. Astrophys. 34, 419 (1996). https://doi.org/10.1146/annurev.astro.34.1.419

94. B. Paczyński, Astrophys. J. Lett. 494(1), L23 (1998). https://doi. org/10.1086/311153

95. M. Miyamoto, Y. Yoshii, Astron. J. 110, 1427 (1995). https://doi. org/10.1086/117616

96. A.F. Boden, M. Shao, D. Van Buren, Astrophys. J. 502(2), 538 (1998). https://doi.org/10.1086/305913

97. Y. Jeong, C. Han, S.H. Park, Astrophys. J. 511(2), 569 (1999). https://doi.org/10.1086/306723

98. A.O. Petters, Mon. Not. R. Astron. Soc. 338, 457 (2003). https:// doi.org/10.1046/j.1365-8711.2003.06065.x

99. F. Peißker, A. Eckart, M. Zajaček, B. Ali, M. Parsa, Astrophys. J. 899(1), 50 (2020). https://doi.org/10.3847/1538-4357/ab9c1c

100. F. Peißker, A. Eckart, M. Parsa, Astrophys. J. 889(1), 61 (2020). https://doi.org/10.3847/1538-4357/ab5afd

101. F. Peißker, A. Eckart, B. Ali, Astrophys. J. 918(1), 25 (2021). https://doi.org/10.3847/1538-4357/ac0efc

102. M.A. Walker, Astrophys. J. 453, 37 (1995). https://doi.org/10. $1086 / 176367$

103. M. Dominik, K.C. Sahu, Astrophys. J. 534, 213 (2000). https:// doi.org/10.1086/308716

104. GRAVITY Collaboration, Astron. Astrophys. 625, L10 (2019). https://doi.org/10.1051/0004-6361/201935656

105. J. Ibanez, Astron. Astrophys. 124, 175 (1983)

106. I. Bray, Phys. Rev. D 34, 367 (1986). https://doi.org/10.1103/ PhysRevD.34.367

107. S.A. Klioner, Sov. Astron. 35, 523 (1991)

108. J.F. Glicenstein, Astron. Astrophys. 343, 1025 (1999)

109. M. Sereno, F. De Luca, Phys. Rev. D 74(12), 123009 (2006). https://doi.org/10.1103/PhysRevD.74.123009

110. M.C. Werner, A.O. Petters, Phys. Rev. D 76(6), 064024 (2007). https://doi.org/10.1103/PhysRevD.76.064024

111. M. Sereno, F. De Luca, Phys. Rev. D 78(2), 023008 (2008). https:// doi.org/10.1103/PhysRevD.78.023008

112. A.B. Aazami, C.R. Keeton, A.O. Petters, J. Math. Phys. 52(9), 092502 (2011). https://doi.org/10.1063/1.3642614

113. A.B. Aazami, C.R. Keeton, A.O. Petters, J. Math. Phys. 52(10), 102501 (2011). https://doi.org/10.1063/1.3642616

114. G. He, W. Lin, Int. J. Mod. Phys. D 23, 1450031 (2014). https:// doi.org/10.1142/S021827181450031X

115. G. He, C. Jiang, W. Lin, Int. J. Mod. Phys. D 23, 1450079 (2014). https://doi.org/10.1142/S0218271814500795

116. X.M. Deng, Int. J. Mod. Phys. D 24, 1550056 (2015). https://doi. org/10.1142/S021827181550056X

117. G.S. He, W.B. Lin, Res. Astron. Astrophys. 15, 646 (2015). https:// doi.org/10.1088/1674-4527/15/5/003

118. X.M. Deng, Int. J. Mod. Phys. D 25, 1650082 (2016). https://doi. org/10.1142/S0218271816500826

119. G. He, W. Lin, Phys. Rev. D 93(2), 023005 (2016). https://doi. org/10.1103/PhysRevD.93.023005

120. G. He, W. Lin, Phys. Rev. D 94(6), 063011 (2016). https://doi. org/10.1103/PhysRevD.94.063011

121. G. He, W. Lin, Class. Quantum Gravity 34(10), 105006 (2017). https://doi.org/10.1088/1361-6382/aa691d

122. W. Li, Z. Feng, X. Zhou, X. Mu, G. He, Int. J. Mod. Phys. D 30(9), 2150067 (2021). https://doi.org/10.1142/S021827182150067X 
123. S.E. Gralla, Phys. Rev. D 103, 024023 (2021). https://doi.org/10. 1103/PhysRevD.103.024023

124. S.E. Gralla, D.E. Holz, R.M. Wald, Phys. Rev. D 100(2), 024018 (2019). https://doi.org/10.1103/PhysRevD.100.024018

125. M.D. Johnson, A. Lupsasca, A. Strominger, G.N. Wong, S. Hadar, D. Kapec, R. Narayan, A. Chael, C.F. Gammie, P. Galison, D.C.M. Palumbo, S.S. Doeleman, L. Blackburn, M. Wielgus, D.W. Pesce, J.R. Farah, J.M. Moran, Sci. Adv. 6(12), eaaz1310 (2020). https:// doi.org/10.1126/sciadv.aaz1310

126. S.E. Gralla, A. Lupsasca, Phys. Rev. D 102, 124003 (2020). https://doi.org/10.1103/PhysRevD.102.124003

127. S.E. Gralla, A. Lupsasca, D.P. Marrone, Phys. Rev. D 102, 124004 (2020). https://doi.org/10.1103/PhysRevD.102.124004

128. M. De Laurentis, R. De Rosa, F. Garufi, L. Milano, Mon. Not. R. Astron. Soc. 424, 2371 (2012). https://doi.org/10.1111/j. 1365-2966.2012.21410.x

129. X.M. Deng, Europhys. Lett. 120(6), 60004 (2017). https://doi. org/10.1209/0295-5075/120/60004

130. X.M. Deng, Eur. Phys. J. Plus 132, 85 (2017). https://doi.org/10. 1140/epjp/i2017-11376-1

131. I. De Martino, R. Lazkoz, M. De Laurentis, Phys. Rev. D 97(10), 104067 (2018). https://doi.org/10.1103/PhysRevD.97.104067

132. M. De Laurentis, I. De Martino, R. Lazkoz, Phys. Rev. D 97(10), 104068 (2018). https://doi.org/10.1103/PhysRevD.97.104068

133. M. De Laurentis, I. De Martino, R. Lazkoz, Eur. Phys. J. C 78(11), 916 (2018). https://doi.org/10.1140/epjc/s10052-018-6401-0

134. T.Y. Zhou, Y. Xie, Eur. Phys. J. C 80(11), 1070 (2020). https:// doi.org/10.1140/epjc/s10052-020-08661-w
135. W. Li, B. Yang, C. Ma, X. Zhou, Z. Feng, G. He, Mod. Phys. Lett. A 36(22), 2150164 (2021). https://doi.org/10.1142/ S0217732321501649

136. J. Levin, G. Perez-Giz, Phys. Rev. D 77(10), 103005 (2008). https://doi.org/10.1103/PhysRevD.77.103005

137. J. Levin, R. Grossman, Phys. Rev. D 79(4), 043016 (2009). https:// doi.org/10.1103/PhysRevD.79.043016

138. J. Levin, G. Perez-Giz, Phys. Rev. D 79(12), 124013 (2009). https://doi.org/10.1103/PhysRevD.79.124013

139. J. Levin, Class. Quantum Gravity 26(23), 235010 (2009). https:// doi.org/10.1088/0264-9381/26/23/235010

140. V. Misra, J. Levin, Phys. Rev. D 82(8), 083001 (2010). https:// doi.org/10.1103/PhysRevD.82.083001

141. B. Gao, X.M. Deng, Ann. Phys. 418, 168194 (2020). https://doi. org/10.1016/j.aop.2020.168194

142. X.M. Deng, Eur. Phys. J. C 80(6), 489 (2020). https://doi.org/10. 1140/epjc/s10052-020-8067-7

143. X.M. Deng, Phys. Dark Univ. 30, 100629 (2020). https://doi.org/ 10.1016/j.dark.2020.100629

144. H.Y. Lin, X.M. Deng, Phys. Dark Univ. 31, 100745 (2021). https:// doi.org/10.1016/j.dark.2020.100745

145. B. Gao, X.M. Deng, Eur. Phys. J. C 81(11), 983 (2021). https:// doi.org/10.1140/epjc/s10052-021-09782-6

146. B. Gao, X.M. Deng, Mod. Phys. Lett. A 36(33), 2150237 (2021). https://doi.org/10.1142/S0217732321502370

147. H.Y. Lin, X.M. Deng, Eur. Phys. J. Plus 137(2), 176 (2022). https://doi.org/10.1140/epjp/s13360-022-02391-6 